\title{
A Stroll through the Loop-Tree Duality
}

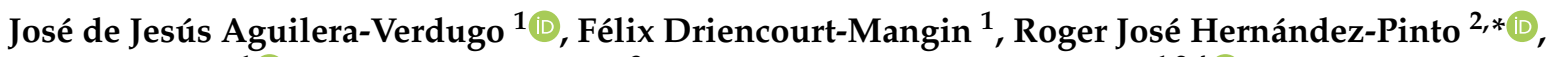 \\ Judith Plenter ${ }^{1}\left(\mathbb{D}\right.$, Renato Maria Prisco ${ }^{3}$, Norma Selomit Ramírez-Uribe ${ }^{1,2,4}{ }^{\oplus}$, Andrés Ernesto Rentería-Olivo ${ }^{1}{ }^{\circledR}$, \\ Germán Rodrigo ${ }^{1} \mathbb{D}$, German Sborlini ${ }^{1,5, *} \mathbb{D}$, William Javier Torres Bobadilla $\left.{ }^{6} \mathbb{(}\right)$ and Francesco Tramontano ${ }^{3}(\mathbb{D})$
}

1 Instituto de Física Corpuscular, Universitat de València-Consejo Superior de Investigaciones Científicas, Parc Científic, E-46980 Paterna, Spain; jesus.aguilera@ific.uv.es (J.d.J.A.-V.); felixdrima@gmail.com (F.D.-M.); plenter@ific.uv.es (J.P.); selomit@ific.uv.es (N.S.R.-U.); andres.renteria@ific.uv.es (A.E.R.-O.); german.rodrigo@csic.es (G.R.)

2 Facultad de Ciencias Físico-Matemáticas, Universidad Autónoma de Sinaloa, Ciudad Universitaria, 80000 Culiacán, Mexico

3 Dipartimento di Fisica, Università di Napoli Federico II and INFN, Sezione di Napoli, I-80126 Napoli, Italy; renatomaria.prisco@unina.it (R.M.P.); francesco.tramontano@na.infn.it (F.T.)

4 Facultad de Ciencias de la Tierra y el Espacio, Universidad Autónoma de Sinaloa, Ciudad Universitaria, 80000 Culiacán, Mexico

5 Deutsches Elektronen-Synchrotron, DESY, Platanenallee 6, D-15738 Zeuthen, Germany

6 Max-Planck-Institut für Physik, Werner-Heisenberg-Institut, Föhringer Ring 6, D-80805 München, Germany; torres@mpp.mpg.de

check for

updates

Citation: Aguilera-Verdugo, J.d.J.; Driencourt-Mangin, F.; Hernández-Pinto, R.J.; Plenter, J.; Prisco, R.M.; Ramírez-Uribe, N.S.; Rentería-Olivo, A.E.; Rodrigo, G.; Sborlini, G.; Torres Bobadilla, W.J.; et al. A Stroll through the Loop-Tree Duality. Symmetry 2021, 13, 1029. https://doi.org/10.3390/sym13061029

Academic Editors: Zoltán Trócsányi, Adam Kardos and Giuseppe Bevilacqua

\section{Received: 29 April 2021}

Accepted: 7 June 2021

Published: 8 June 2021

Publisher's Note: MDPI stays neutral with regard to jurisdictional claims in published maps and institutional affiliations.

Copyright: (c) 2021 by the authors. Licensee MDPI, Basel, Switzerland. This article is an open access article distributed under the terms and conditions of the Creative Commons Attribution (CC BY) license (https:// creativecommons.org/licenses/by/ $4.0 /)$.
* Correspondence: roger@uas.edu.mx (R.J.H.-P.); german.sborlini@desy.de (G.S.)

\begin{abstract}
The Loop-Tree Duality (LTD) theorem is an innovative technique to deal with multi-loop scattering amplitudes, leading to integrand-level representations over a Euclidean space. In this article, we review the last developments concerning this framework, focusing on the manifestly causal representation of multi-loop Feynman integrals and scattering amplitudes, and the definition of dual local counter-terms to cancel infrared singularities.
\end{abstract}

Keywords: Feynman integrals; multi-loop calculations; perturbative QFT; higher orders

\section{Introduction}

The most successful description of the microscopic structure of Nature is currently given by the Standard Model (SM), which is based on quantum field theories (QFT) with specific gauge symmetries. The strong interplay between experiment and theory relies on confronting precise predictions with accurate data. In the context of high-energy particle physics, the Large Hadron Collider (LHC) and the planned future colliders [1-8] will keep on reducing the experimental uncertainties, forcing to reach a compatible accuracy level from the theory side. However, there are huge bottlenecks that prevent straightforward calculations.

Due to their complexity, exact solutions of QFTs are not available for generic scattering processes. Thus, extracting predictions from theory requires the use of approximations, whose applicability is restricted to specific situations. Although lattice methods are reliable in the low-energy regime, perturbation theory is the most suitable strategy to tackle the phenomenological description of particle collisions at high-energies. Even if the perturbative approach reduces the original calculation to a series expansion in the interaction couplings, adding higher orders is far from trivial due to the presence of complicated phase-space and Feynman loop integrals. Moreover, these objects feature numerical instabilities originated in threshold configurations, as well as other singularities that cancel only after putting all the contributions together.

In the last 40 years, a huge effort has been done to develop new methodologies for an efficient calculation of physical observables at higher orders. As a first step, proper regularization methods have been applied to make explicit the singular structures of these 
objects. One of the customary choices is Dimensional Regularization (DREG), which consists of modifying the number of space-time dimensions to achieve integrability [9-12]. However, in the context of QFT, changing the number of space-time dimensions leads to definition problems, such as the $\gamma_{5}[13,14]$, and prevents a straightforward numerical implementation. For this and other technical reasons, there is an ongoing effort in the high-energy physics community to develop new strategies that locally regularize QFT while keeping the standard four dimensions of the space-time [15-20].

Once the Feynman amplitudes and phase-space integrals are properly regularized, we need to compute them and combine all the ingredients. On the one hand, this requires calculation of multi-loop multi-leg Feynman integrals. Several techniques are currently available for this purpose: Feynman parametrization, Mellin-Barnes transformations, IBP identities [21,22], sector decomposition [23-26], semi-numerical approaches [27-29], among others [30]. Very recently, algebraic geometry [31-36] was used to develop multiloop integrand reduction algorithms [37-44], and to explore alternative representations of Feynman integrals [45,46]. Additionally, unitarity-based methods [47-50] and intersection theory [51-54] are being studied to improve the reduction to master integrals and their subsequent evaluation.

On the other hand, loop contributions must be combined with real-emission corrections and suitable counter-terms to cancel the infrared (IR) and ultraviolet (UV) singularities, leading to a reliable prediction of the physical cross-sections and differential distributions. There are several strategies to achieve this cancellation, most of them based on the subtraction methods [55-57]. Generally speaking, these frameworks involve adding and subtracting counter-terms which locally cancel the IR behavior of the real part, but at the same time they are easy enough to be integrated analytically and subtracted from the virtual contribution. There are several variations of this approach: dipole subtraction $[57,58]$, $q_{T}$ subtraction/resummation [59,60], antenna subtraction [61], colorful subtraction [62,63], among others. Additionally, there are alternatives that rely on a different way to achieve the cancellation of singularities, such as the n-jettiness $[64,65]$ or the local analytic subtraction frameworks [66,67].

Besides those methods, a novel strategy to tackle, simultaneously, the efficient calculation of loop integrals and physical observables was developed. This framework is based on the Loop-Tree Duality (LTD) theorem [68-71], which opens loops into trees and recasts virtual states into configurations that resemble real-radiation processes. From the mathematical point of view, the LTD transforms the integration domain of loop integrals into a Euclidean space. In this way, a more intuitive understanding of the regions responsible for the singular structure of the loop integrals emerges [72]. This knowledge can be used to explore novel techniques pointing towards more efficient numerical implementations [73,74], integrand-level simplifications through asymptotic expansions [75-77] and unveiling the universal structures of scattering amplitudes at higher orders [78-81]. Moreover, the LTD provides the perfect framework to handle cross-section calculations in a fully unified way. Since the dual representation of loop integrals is defined in Euclidean domains, the virtual and real contributions can be directly combined at integrand level to achieve a fully local cancellation of IR singularities. This is the so-called Four-Dimensional Unsubtraction (FDU) approach [82-85] that profits from a local cancellation of singularities which makes it possible to bypass additional regulators (such as DREG). Additionally, this formalism allows us to write local UV counter-terms that exactly reproduce the expected results in conventional renormalization schemes $[80,81,84,85]$, as well as fully local IR dual counter-terms [86].

During the last two years, we have explored new features of the LTD approach. In particular, we discovered that a more general analysis of the singular structure of multi-loop multi-leg amplitudes is possible. By inquiring into the physical and anomalous thresholds of one and two-loop amplitudes [87], we proved that it was possible to remove non-physical singularities and retain only those contributions compatible with causality. Several studies about the causal structure of scattering amplitudes are available in the literature, using 
different techniques [88-91]. In Ref. [92], we presented for the first time a manifestly causal integrand-level representation inspired by the LTD theorem. This was applied to remove unphysical threshold singularities and obtain causal integrand-level representations of several topological families of multi-loop multi-leg Feynman integrals [92-99]. Moreover, all-order causal formulae were obtained using novel algebraic relations [100], leading to an automatized framework to implement these calculations [101]. A purely geometrical interpretation [102] was also developed, showing a complementary approach to tackle the causal structure of multi-loop multi-leg scattering amplitudes and Feynman integrals. Very recently, this last strategy has been also studied in the context of quantum algorithms [103], to explore novel techniques to speed up the calculations in high-energy physics.

The purpose of this review is to give a brief summary of the latest developments regarding the LTD-based methods. To ease the presentation, it is organized in two parts. In the first one, starting in Section 2, we settle the notation of the LTD formalism. Special emphasis is put on the manifestly causal representation of loop integrands in the Euclidean space, as well as its derivation from Cauchy's residue theorem. The basis for a rigorous mathematical treatment of the nested residue strategy is given in Section 3. Then, we discuss the advantages of the Euclidean representation of loop integrands. In Section 4, we focus on the asymptotic expansion, while we discuss how to obtain compact causal formulae for generic topological families of multi-loop multi-leg Feynman integrals and scattering amplitudes in Sections 5 and 6. In Section 7, a few words are given to explain novel developments suggesting a deep connection among graph theory, algebra and the causal structure of multi-loop scattering amplitudes.

After that, we start the second part of the review, where we center into the computation of physical observables exploiting the LTD approach. In Section 8 we discuss the basis of the FDU formalism, showing a physical example. Then, in Section 9, the construction of local counter-terms for cancelling IR singularities is presented with more general examples, including $\gamma^{*} \rightarrow 3$ jets at NLO. Finally, we present the conclusions and future research directions in Section 10.

\section{Causality within the LTD Formulation}

Among the several advantages of the LTD formalism, we have recently explored the manifestly causal representations. This constitutes a big step towards an efficient numerical implementation of this approach, since spurious non-physical and noncausal singularities are completely avoided. In the following, we will introduce some useful notation and describe the basis of the formalism. In particular, we will explain how the LTD computation through the nested residue strategy leads to the causal representations of different topological families to all-loop orders.

\subsection{Dual Scattering Amplitudes}

A generic L-loop scattering amplitude with $N$ external legs $\left\{p_{j}\right\}_{N}$, and $n$ sets of internal lines, each set being defined by the specific dependence on the loop momenta, is written as

$$
\mathcal{A}_{N}^{(L)}(1, \ldots, n)=\int_{\ell_{1}, \ldots, \ell_{L}} \mathcal{A}_{F}^{(L)}(1, \ldots, n)
$$

with

$$
\mathcal{A}_{F}^{(L)}(1, \ldots, n)=\mathcal{N}\left(\left\{\ell_{s}\right\}_{L},\left\{p_{j}\right\}_{N}\right) \times G_{F}(1, \ldots, n) .
$$

This corresponds to an integral in the Minkowski space of the L-loop momenta, $\left\{\ell_{S}\right\}_{L}$, involving the product of Feynman propagators, $G_{F}\left(q_{i}\right)=\left(q_{i}^{2}-m_{i}^{2}+\imath 0\right)^{-1}$, and numerators, $\mathcal{N}\left(\left\{\ell_{S}\right\}_{L},\left\{p_{j}\right\}_{N}\right)$, given by the Feynman rules of the theory considered. The $d$-dimensional integration measure in DREG reads

$$
\int_{\ell_{s}}=-\imath \mu^{4-d} \int \mathrm{d}^{d} \ell_{s} /(2 \pi)^{d}
$$

and the usual Feynman propagator for a single particle is 


$$
G_{F}\left(q_{i_{s}}\right)=\frac{1}{q_{i_{s}, 0}^{2}-\left(q_{i_{s}, 0}^{(+)}\right)^{2}},
$$

where

$$
q_{i_{s}, 0}^{(+)}=\sqrt{\mathbf{q}_{i_{s}}^{2}+m_{i_{s}}^{2}-\imath 0},
$$

is the positive on-shell energy of the loop momentum $q_{i_{s}}$, written in terms of its spatial components $\mathbf{q}_{i_{s}}$, its mass $m_{i_{s}}$, and the Feynman's infinitesimal imaginary prescription $\imath 0$. Then, we introduce the shorthand notation

$$
G_{F}(1, \ldots, n)=\prod_{i_{s} \in 1 \cup \ldots \cup n}\left(G_{F}\left(q_{i_{s}}\right)\right)^{a_{i_{s}}},
$$

to express the product of Feynman propagators of one set or the union of several sets. In the former formula, $s$ represents the set of all internal propagators with internal momenta $q_{i_{s}}=\ell_{s}+k_{i_{s}}$, that depend on the loop momentum, $\ell_{s}$, or a specific linear combination of loop momenta, and a combination of external momenta $k_{i_{s}}$, with $i_{s} \in s$ and $a_{i_{s}}$ arbitrary powers.

The LTD representation for scattering amplitudes is obtained by the iterative application of the Cauchy's residue theorem (CRT) integrating out one degree of freedom for each loop momentum, and closing the Cauchy contour from below the real axis, selecting the poles with negative imaginary part in the complex plane of the loop momentum. This results in the modification of the infinitesimal complex prescription of the Feynman propagators $[68,69]$ that needs to be considered carefully to preserve causality. Then, starting from Equation (1), we set on-shell the propagators that depend on the first loop momentum, $q_{i_{1}}$, and define

$$
\mathcal{A}_{D}^{(L)}(1 ; 2, \ldots, n) \equiv \sum_{i_{1} \in 1} \operatorname{Res}\left(\mathcal{A}_{F}^{(L)}(1, \ldots, n), \operatorname{Im}\left(q_{i_{1}, 0}<0\right)\right),
$$

where taking the residue is equivalent to integrating out the energy component of the loop momenta. Now, we construct the nested residue iterating until the $r$-th set as,

$$
\mathcal{A}_{D}^{(L)}(1, \ldots, r ; r+1, \ldots, n)=\sum_{i_{s} \in S} \operatorname{Res}\left(\mathcal{A}_{D}^{(L)}(1, \ldots, r-1 ; r, \ldots, n), \operatorname{Im}\left(q_{i_{s}, 0}<0\right)\right) .
$$

All sets before the semicolon contain one propagator that has been set on-shell and are linearly independent, while all the remaining propagators are kept off-shell. Thus, this representation is equivalent to open the loop amplitude to nondisjoint trees. Finally, the integration measure after integrating out the energy component is modified according to

$$
\int_{\vec{\ell}_{s}} \equiv-\mu^{d-4} \int \frac{\mathrm{d}^{d-1} \ell_{s}}{(2 \pi)^{d-1}}
$$

transforming the $d$-dimensional Minkowski space into a $(d-1)$-dimensional Euclidean one.

\subsection{Multi-Loop Topologies through the LTD}

By the properties obtained from the nested residues, Equation (8), we can construct multi-loop amplitudes from the LTD representation which are compact and manifestly causal to all orders. The first topology under consideration is called Maximal Loop Topology (MLT), and is characterized by $L+1$ momentum sets where the momenta of the first $L$ sets depend on one single loop momentum

$$
q_{i_{s}}=\ell_{s}+k_{i_{s}}
$$


with $s \in\{1, \ldots, L\}$. The momenta of the extra set, $L+1$, are given by a linear combination of all the loop momenta, namely $q_{i_{L+1}}=-\sum_{s=1}^{L} \ell_{s}+k_{i_{L+1}}$. Here, $k_{i_{s}}$ and $k_{i_{L+1}}$ denote linear combinations of external momenta. The LTD representation of the MLT topology, displayed in Figure 1a, is presented in a compact and symmetric form by evaluating the nested residue of Equation (8), which leads to

$$
\mathcal{A}_{\mathrm{MLT}}^{(L)}(1, \ldots, L+1)=\int_{\vec{\ell}_{1}, \ldots, \vec{\ell}_{L}} \sum_{i=1}^{L+1} \mathcal{A}_{D}^{(L)}(1, \ldots, i-1, \overline{i+1}, \ldots, \overline{L+1} ; i),
$$

where the bars within the integrand indicate a reversal of momentum flow, $q_{\bar{i}_{s}}=-q_{i_{s}}$, which is needed to preserve causality, and is equivalent to select the on-shell modes with negative energy of the original momentum flow. Each term of the sum in the integrand of Equation (11) contains one set $i$ with all its propagators off-shell, while the remaining $L$ sets contain a single on-shell propagator each; a necessary condition to open multi-loop amplitudes into disjoint trees. The dual representation of Equation (11) becomes singular when one or more off-shell propagators eventually become on-shell, generating a disjoint tree dual subamplitude.

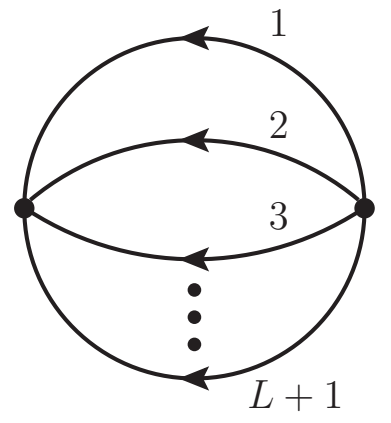

a) MLT

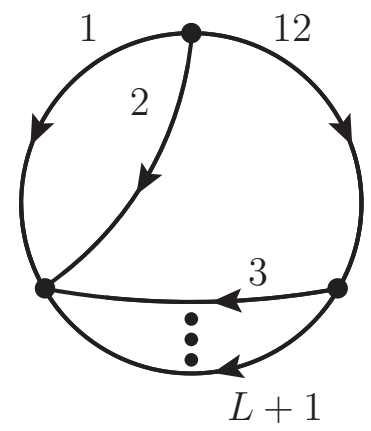

b) NMLT

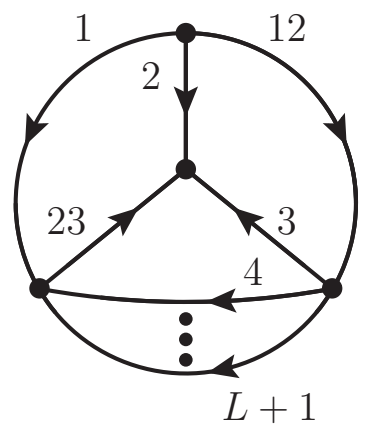

c) $\mathrm{N}^{2} \mathrm{MLT}$

Figure 1. Graphical representations of Maximal Loop Topology (MLT), Next-to-Maximal Loop Topology (NMLT) and Next-to-Next-to-Maximal Loop Topology $\left(\mathrm{N}^{2} \mathrm{MLT}\right)$. External momenta are not shown.

It is worth mentioning that the LTD representation exhibits an interesting structure when all the contributions are added together. For example, in the case of a MLT configuration with one propagator in each loop set and one incoming and outgoing momentum, we obtain

$$
\mathcal{A}_{\mathrm{MLT}}^{(L)}(1, \ldots, L+1)=-\int_{\vec{\ell}_{1}, \ldots, \vec{\ell}_{L}} \frac{1}{x_{L+1}}\left(\frac{1}{\lambda_{1}^{-}}+\frac{1}{\lambda_{1}^{+}}\right),
$$

with

$$
x_{L+k}=2^{L+k} \prod_{i=1}^{L+k} q_{i, 0}^{(+)}, \quad \text { and } \quad \lambda_{1}^{ \pm}=\sum_{i=1}^{L+1} q_{i, 0}^{(+)} \pm k_{0, L+1} .
$$

This expression is free of unphysical singularities, and written in terms of the so-called causal propagators, $\lambda_{1}^{ \pm}$. The causal propagators encode all the possible physical singularities that might occur. Both $\lambda_{1}^{ \pm}$in the integrand of Equation (12) are associated with physical thresholds, as shown in Figure 2. Additionally, Equation (12) is independent of the initial momentum configuration in the Feynman representation. 


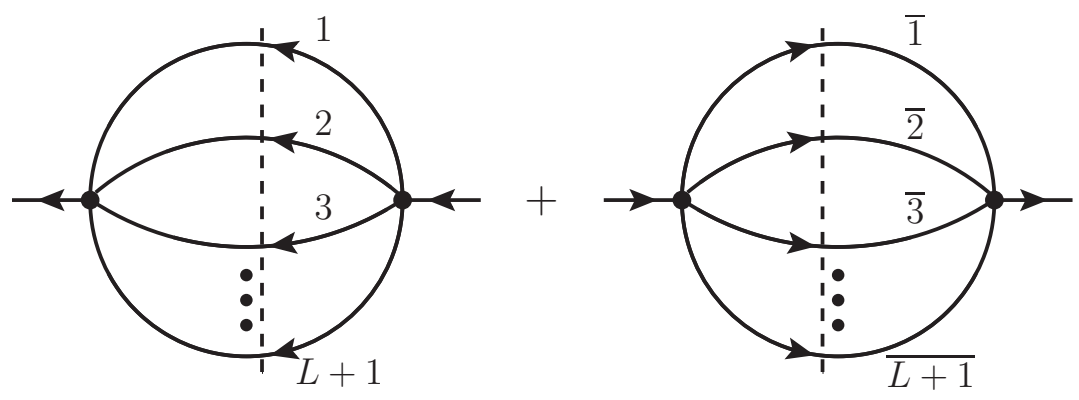

Figure 2. Representation of the causal structure of MLT topologies.

Going a step further in the topology complexity, we consider a topology containing one extra set of momenta that depends on the sum of two-loop momenta, $q_{i_{12}}=-\left(\ell_{1}+\right.$ $\left.\ell_{2}\right)+k_{i_{12}}$, denoted as 12. This configuration, as can be appreciated in Figure 1b, is called Next-to-Maximal Loop Topology (NMLT), characterized by $L+2$ sets of propagators, with each set categorized by the dependence on a specific loop momentum or a linear combination of the $L$ independent loop momenta. The LTD representation for the NMLT is given by the compact and factorized expression

$$
\begin{aligned}
\mathcal{A}_{\mathrm{NMLT}}^{(L)}(1, \ldots, L+2) & =\mathcal{A}_{\mathrm{MLT}}^{(2)}(1,2,12) \otimes \mathcal{A}_{\mathrm{MLT}}^{(L-2)}(3, \ldots, L+1) \\
& +\mathcal{A}_{\mathrm{MLT}}^{(1)}(1,2) \otimes \mathcal{A}^{(0)}(12) \otimes \mathcal{A}_{\mathrm{MLT}}^{(L-1)}(\overline{3}, \ldots, \overline{L+1}),
\end{aligned}
$$

involving convolutions of MLT subtopologies. The singular structure of the factorized subtopologies determine the causal thresholds and infrared singularities. For example, taking $L=3$, i.e., three loops, the convolutions within the NMLT configuration are interpreted as

$$
\begin{aligned}
\mathcal{A}_{\mathrm{MLT}}^{(2)}(1,2,12) \otimes \mathcal{A}_{\mathrm{MLT}}^{(1)}(3,4) & =\int_{\vec{\ell}_{1}, \vec{\ell}_{2}, \vec{\ell}_{3}}\left(\mathcal{A}_{D}^{(3)}(\overline{2}, \overline{12}, \overline{4} ; 1,3)+\mathcal{A}_{D}^{(3)}(1, \overline{12}, \overline{4} ; 2,3)\right. \\
& \left.+\mathcal{A}_{D}^{(3)}(1,2, \overline{4} ; 12,3)+(\overline{4} \leftrightarrow 3)\right),
\end{aligned}
$$

and

$$
\begin{aligned}
\mathcal{A}_{\mathrm{MLT}}^{(1)}(1,2) \otimes \mathcal{A}^{(0)}(12) \otimes \mathcal{A}_{\mathrm{MLT}}^{(2)}(\overline{3}, \overline{4}) & =\int_{\vec{\ell}_{1}, \vec{\ell}_{2}, \vec{\ell}_{3}}\left(\mathcal{A}_{D}^{(3)}(\overline{2}, \overline{3}, \overline{4} ; 1,3)\right. \\
& \left.+\mathcal{A}_{D}^{(3)}(1, \overline{3}, \overline{4} ; 2,12)\right),
\end{aligned}
$$

where the sets after the semicolon are put off-shell.

Following the previous procedure, we also presented in Ref. [92] the LTD representation for the Next-to-Next-to-Maximal Loop Topology ( $\left.\mathrm{N}^{2} \mathrm{MLT}\right)$, characterized by $L+3$ sets of propagators and depicted in Figure 1c. Again, factorized formulae for the dual representation are obtained, namely

$$
\begin{aligned}
\mathcal{A}_{\mathrm{N} 2 \mathrm{MLT}}^{(L)}(1, \ldots, L+3,12,23) & =\mathcal{A}_{\mathrm{NMLT}}^{(L)}(1,2,3,12,23) \otimes \mathcal{A}_{\mathrm{MLT}}^{(L-3)}(4, \ldots, L+1) \\
& +\mathcal{A}_{\mathrm{MLT}}^{(2)}(1 \cup 23,2,3 \cup 12) \otimes \mathcal{A}_{\mathrm{MLT}}^{(L-2)}(\overline{4}, \ldots, \overline{L+1}),
\end{aligned}
$$

showing a recursive construction similar to the one reported for the NMLT configurations. Expanding the first term on the right-hand side of Equation (17) into its corresponding subtopologies, we have

$$
\begin{aligned}
\mathcal{A}_{\mathrm{NMLT}}^{(L)}(1,2,3,12,23) & =\mathcal{A}_{\mathrm{MLT}}^{(2)}(1,2,12) \otimes \mathcal{A}_{\mathrm{MLT}}^{(3)}(3,23) \\
& +\int_{\vec{\ell}_{1}, \vec{\ell}_{2}, \vec{\ell}_{3}}\left(\mathcal{A}_{D}^{(3)}(1, \overline{3}, \overline{23} ; 2,12)+\mathcal{A}_{D}^{(3)}(\overline{12}, 3,23 ; 1,2)\right),
\end{aligned}
$$

where the last two terms of the right-hand side in the integrand remain fixed by the condition that the sets $(2,3,23)$ cannot generate a disjoint subtree. We can observe that the second term in Equation (17), contain a two-loop subtopology involving five sets of 
momenta grouped into three sets. Therefore, the propagators in the set 1 and 23 cannot be simultaneously off shell.

Noteworthy, there are very compact explicit formulae for the NMLT and $\mathrm{N}^{2} \mathrm{MLT}$ configurations which make use of the causal propagators. In Section 5, we will enter into more detail, and provide a nice conceptual interpretation of the manifestly causal dual representation in terms of entangled thresholds.

\section{Mathematical Properties of the Nested Residues}

Generic scattering amplitudes are defined by integrals of rational functions. As already explained in Section 2, the multi-loop LTD framework is based on the CRT and, in this section, the formal foundations for an L-loop Feynman diagram and some of their immediate consequences are explained. As discussed in Ref. [97], iterated residues can be easily computed taking into account the quadratic structure of Feynman propagators. Let us start with the space $\mathbb{C}^{\left(\mathbb{R}^{L}\right)}$ of the functions with domain $\mathbb{R}^{L}$ and co-domain $\mathbb{C}$. If the natural inclusion of $\mathbb{R}$ into $\mathbb{C}$ is denoted by $i$, then the iterated residues are defined as the recursive application of the functor - Res $\circ i: \mathbb{C}^{\left(\mathbb{R}^{n}\right)} \rightarrow \mathbb{C}^{\left(\mathbb{R}^{n-1}\right)}$, where Res represents the residue of the argument for all its negative imaginary part poles and the minus sign appears in agreement with CRT as the integration is performed clockwise.

For the computation of multi-loop Feynman integrals and scattering amplitudes, internal momenta are given as linear combinations of external and loop momenta. For instance, for the scalar sunrise diagram, the associated integrand has the form

$$
\mathcal{I}(p)=\frac{1}{\left(q_{1,0}^{2}-q_{1,0}^{(+) 2}\right)\left(q_{2,0}^{2}-q_{2,0}^{(+) 2}\right)\left(\left(q_{1,0}+q_{2,0}+k_{3,0}\right)^{2}-q_{3,0}^{(+) 2}\right)}
$$

In this case, the first iteration of the iterated residues can be developed with respect to the variable $q_{1,0}$. This means that we extend $q_{1,0}$ to the complex plane, and as $q_{2,0}$ is still a real variable, the poles are located in the complex plane as shown in Figure 3 , where the dashed line represents the poles $\pm q_{3,0}^{(+)}-q_{2,0}-k_{3,0}$. As they depend on $q_{2,0}$, they can be located at some point along each of the dashed lines.

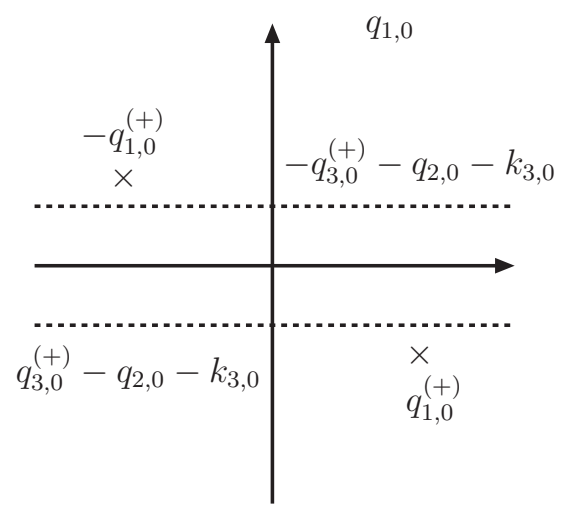

Figure 3. Pole structure of a rational function of two variables.

Then, after the computation of the first iterated residues, we obtain

$$
\begin{aligned}
\operatorname{Res}\left[\mathcal{I}(p),\left\{q_{2,0}, \operatorname{Im}\left(q_{2,0}\right)<0\right\}\right] & =\frac{1}{2 q_{1,0}^{(+)}\left(q_{2,0}^{2}-q_{2,0}^{(+) 2}\right)\left(\left(q_{1,0}^{(+)}+q_{2,0}+k_{3,0}\right)^{2}-q_{3,0}^{(+) 2}\right)} \\
& +\frac{1}{2 q_{3,0}^{(+)}\left(\left(q_{3,0}^{(+)}-q_{2,0}-k_{3,0}\right)^{2}-q_{1,0}^{(+) 2}\right)\left(q_{2,0}^{2}-q_{2,0}^{(+) 2}\right)}
\end{aligned}
$$


The function obtained after the first residue iteration is given as a sum of terms. The next step corresponds to the extension of the variable $q_{2,0}$ to the complex plane. There, the pole structure of each term of Equation (20) is quite similar. This can be represented pictorially as shown in the Figure 4, where the pole structure of each term is presented as a complex plane term. The gray blob in both terms represents the dependence of the imaginary part of the pole $q_{3,0}^{(+)}-q_{2,0}^{(+)}-k_{3,0}$ on the three-momenta $\boldsymbol{q}_{2}$ and $\boldsymbol{q}_{3}$, with no definite imaginary part sign.

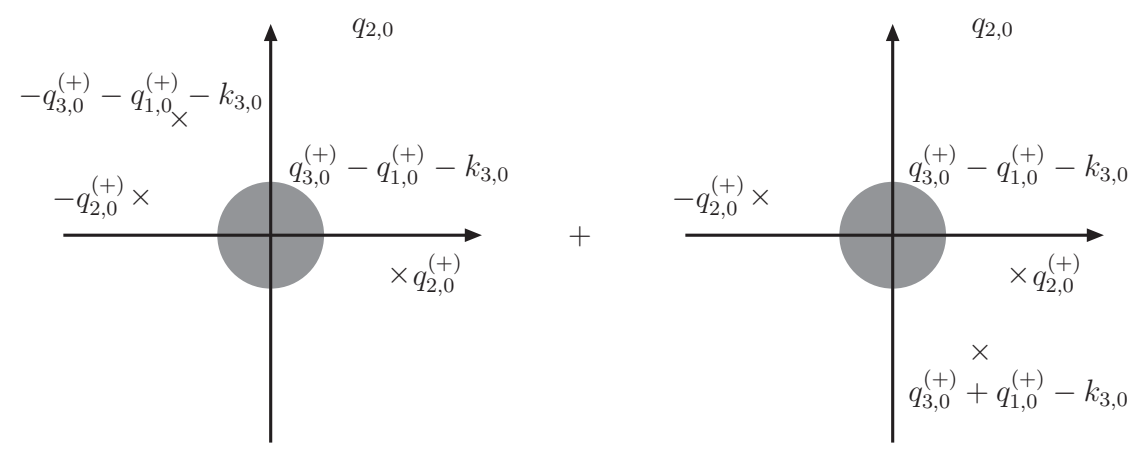

Figure 4. Pole structure of the first residue of a rational function of two variables.

These poles with no definite imaginary part sign are, in general, called displaced poles, and they happen to cancel their contributions to the iterated residues through the relation

$$
\begin{aligned}
& \operatorname{Res}\left(\operatorname{Res}\left(F\left(q_{i, 0}, q_{j, 0}\right),\left\{q_{i, 0}, q_{i, 0}^{(+)}+a_{i}\right\}\right),\left\{q_{j, 0}, q_{k, 0}^{(+)}-q_{i, 0}^{(+)}+a_{i j}-a_{i}\right\}\right) \\
& =-\operatorname{Res}\left(\operatorname{Res}\left(F\left(q_{i, 0}, q_{j, 0}\right),\left\{q_{i, 0}, q_{k, 0}^{(+)}-q_{j, 0}+a_{i j}\right\}\right),\left\{q_{j, 0}, q_{k, 0}^{(+)}-q_{i, 0}^{(+)}+a_{i j}-a_{i}\right\}\right)
\end{aligned}
$$

with

$$
F\left(q_{i, 0}, q_{j, 0}\right)=\frac{P\left(q_{i, 0}, q_{j, 0}\right)}{\left(\left(q_{i, 0}-a_{i}\right)^{2}-q_{i, 0}^{(+) 2}\right)^{\gamma_{i}}\left(\left(q_{i, 0}+q_{j, 0}-a_{i j}\right)^{2}-q_{k, 0}^{(+) 2}\right)^{\gamma_{k}}},
$$

for $a_{i}$ and $a_{i j}$ linear combinations of energy components of external momenta. Due to this cancellation, displaced poles can be ignored in the computation, leading to the concept of nested residues. Furthermore, the final result of the nested residues is independent of the order of the iteration, although the expressions are not identical term by term. A rigorous proof of Equation (21) is shown in Ref. [97].

As a consequence of the nested residue strategy, we can infer a general formula for the causal structure of the MLT diagram (see Figure 1a). This is directly reached applying partial fractions to each term in the nested residue, leading to

$$
G_{F}(1, \ldots, L+1) \rightarrow-\frac{1}{\prod_{i=1}^{L+1} 2 q_{i, 0}^{(+)}}\left(\frac{1}{\sum_{i=1}^{L+1} q_{i, 0}^{(+)}+p_{0}}+\frac{1}{\sum_{i=1}^{L+1} q_{i, 0}^{(+)}-p_{0}}\right)
$$

Additionally, this result can be used for the insertion of an MLT diagram within a more general topology. In this manner, it is possible to consider any MLT insertion as a single propagator whose on-shell energy is the sum of the on-shell energies of all the internal propagators of the MLT.

Furthermore, with the computation of the nested residues, every family of a given topological complexity $k, \mathrm{~N}^{k-1} \mathrm{MLT}$, can be re-expressed as a linear combination of convolutions of lower topological complexity families, as it is for the NMLT and $N^{2}$ MLT, both defined by their amplitudes, 


$$
\begin{gathered}
\mathcal{A}_{\mathrm{NMLT}}^{(L)}(1, \ldots, L+2) \equiv \int_{\ell_{1}, \ldots, \ell_{L}} \mathcal{N}\left(\left\{\ell_{i}\right\}_{L},\left\{p_{j}\right\}_{N}\right) \times G_{F}(1, \ldots, L+1,12), \\
\mathcal{A}_{\mathrm{N}^{2} \mathrm{MLT}}^{(L)}(1, \ldots, L+3) \equiv \int_{\ell_{1}, \ldots, \ell_{L}} \mathcal{N}\left(\left\{\ell_{i}\right\}_{L},\left\{p_{j}\right\}_{N}\right) \times G_{F}(1, \ldots, L+1,12,23) .
\end{gathered}
$$

This decomposition is shown in Figures 5 and 6 for each of these topological families.
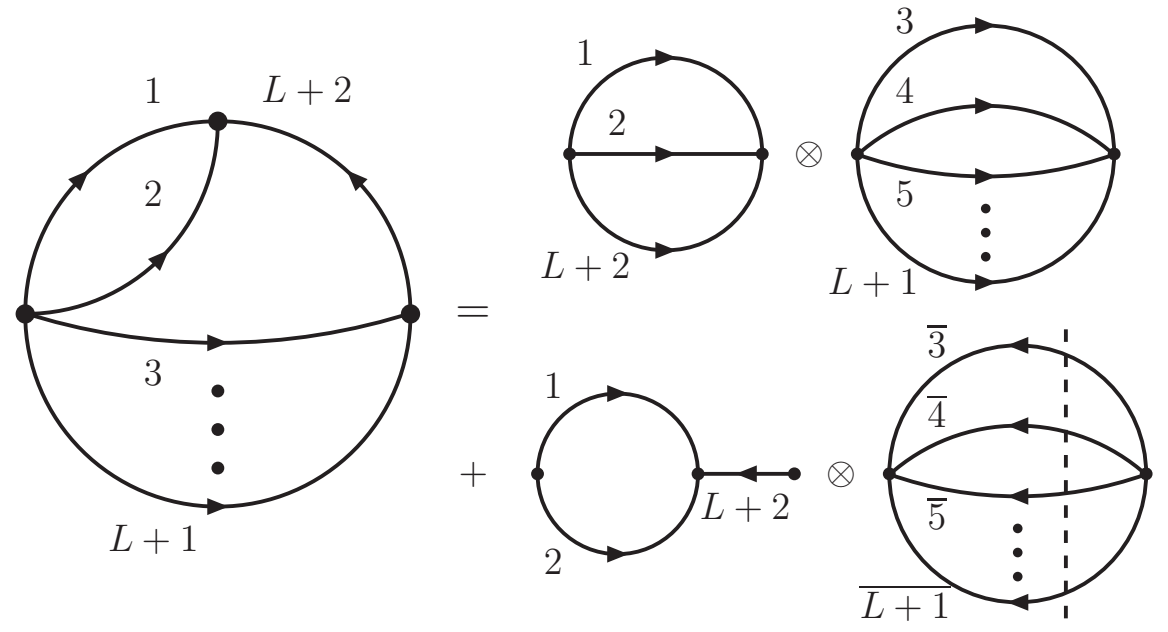

Figure 5. Dual decomposition of $\operatorname{NMLT}(L)$ in terms of loop configurations with lower topological complexity.
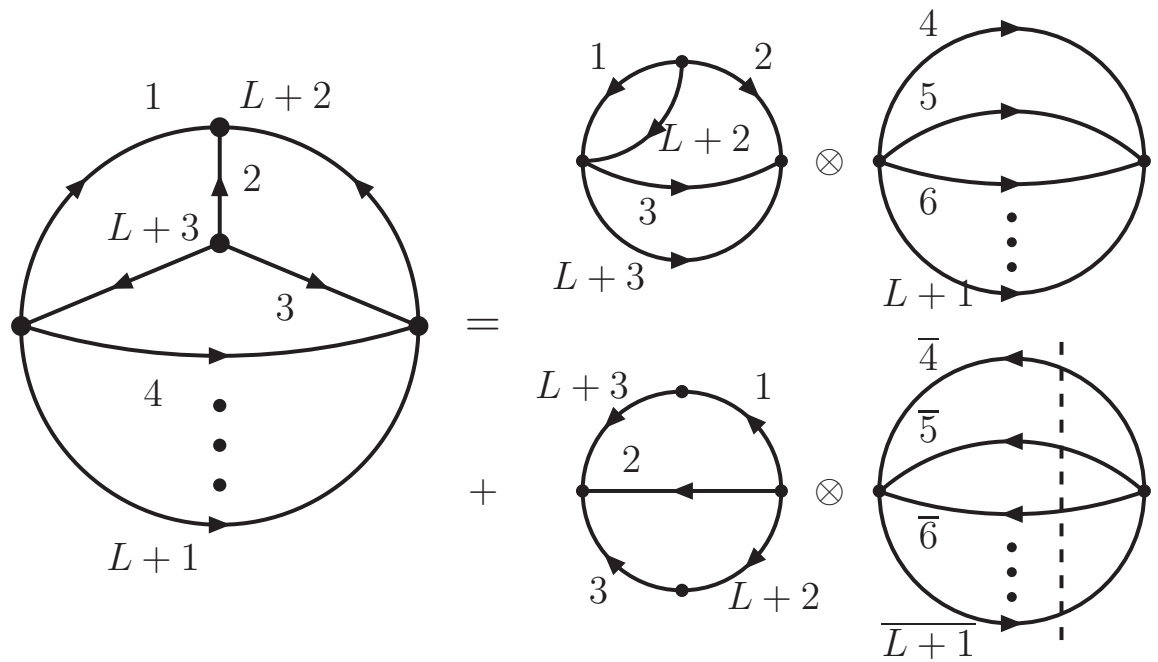

Figure 6. Dual expansion of a $\mathrm{N}^{2} \mathrm{MLT}(L)$ diagram.

It is worth mentioning that the convolution symbol does not represent a pure factorization, as it implies the use of the on-shell conditions to express all the off-shell momenta. The NMLT $(L)$ diagram decomposes into two terms with two MLT diagrams. One of these terms contains an $\operatorname{MLT}(L-2)$ in convolution with an $\operatorname{MLT}(2)$, while the other term contains a convolution of an $\operatorname{MLT}(L-2)$ diagram with all its internal lines on-shell with an MLT(1) diagram and one off-shell momentum. In the case of the $\mathrm{N}^{2} \mathrm{MLT}$ diagram, it is also decomposed into two terms. One of these terms is a convolution of an NMLT(3) diagram with an $\operatorname{MLT}(L-3)$ diagram, and the other term is a convolution of one fully on-shell $\operatorname{MLT}(L-3)$ diagram with an MLT(3) diagram with two external momenta inserted in the 
first and third internal set. These external momenta play a fundamental role in splitting the internal sets into two propagators each. In this manner, the following relations are fulfilled

$$
\begin{aligned}
\operatorname{NMLT}(L) & =\operatorname{MLT}(2) \otimes \operatorname{MLT}(L-2)+G_{F}(L+2) \otimes \operatorname{MLT}(1) \otimes \operatorname{MLT}(L-2), \\
\mathrm{N}^{2} \operatorname{MLT}(L) & =\operatorname{NMLT}(3) \otimes \operatorname{MLT}(L-3)+\operatorname{MLT}(2) \otimes \operatorname{MLT}(L-3),
\end{aligned}
$$

which justifies the factorization relations presented in Section 2.

The topological complexity of the subdiagrams in the convolutions is an additive quantity whose sum coincides with the topological complexity of the original diagram. Recalling that the family of Feynman diagrams with topological complexity $k$ with $L$ loops is $\mathrm{N}^{k-1} \mathrm{MLT}(L)$, for the NMLT(L) has $k_{\mathrm{NMLT}}=2$ and the term in the decomposition with no fully on-shell diagrams is given as the convolution of MLT diagrams (with $k_{\mathrm{MLT}}=1$ ), thus $2 k_{\mathrm{MLT}}=k_{\mathrm{NMLT}}$. For the $\mathrm{N}^{2} \mathrm{MLT}(L)$ it is given $k_{\mathrm{N}^{2} \mathrm{MLT}}=3$, while the term in its decomposition with no fully on-shell diagrams is the convolution of an $\operatorname{NMLT}(L)$ and an $\operatorname{MLT}(L)$ diagram, hence, $k_{\mathrm{NMLT}}+k_{\mathrm{MLT}}=k_{\mathrm{N}^{2} \mathrm{MLT}}$.

These results suggest a factorization formula for each topological complexity family. Indeed, these formulae can be reached through the nested residues and reveal a relation between one topological family with topologies with lower complexity. Within such an approach, every topological family can be expanded by factorizing the MLT diagrams; thus, for any topological family, its causal structure can be expressed following Equation (23). We profited from these structures to derive all-order formulae for $\mathrm{N}^{3} \mathrm{MLT}$ and $\mathrm{N}^{4} \mathrm{MLT}$ families, as explained in Section 6.

\section{Asymptotic Expansions within LTD}

The LTD formalism is straightforwardly applicable to obtain several simplifications of the integrand-level representations of loop amplitudes, such as asymptotic expansions.

The reason asymptotic expansions are an important topic is that they allow the obtaining of analytic expressions, albeit restricted to certain kinematic configurations, for integrals that in their non-expanded form can as of yet not be analytically evaluated. In calculations of physically relevant observables a universally valid analytic expression is indeed not always necessary-be it that the relevant physical effects or the occurrence of computationally relevant artefacts such as soft singularities are restricted to a certain kinematic limit. An example of the former would be the identification of new physics contributions which tend to have unequal effects on different kinematic regimes of an amplitude. When it comes to cancellations between soft singularities and their real-emission counterparts being able to obtain simplified expressions for the divergent limit through asymptotic expansions of the virtual contributions is expected to reduce computation time when ensuring the cancellations during numerical integration.

Since after the application of LTD an amplitude is reduced to an integral over Euclidean three-momenta, the size of the appearing scalar products can be unambiguously be compared to external scales. This provides a good starting point for the development of a well-defined formalism for asymptotic expansions of the integrand. Specific asymptotic expansions in the context of LTD have been presented for the first time for the process $H \rightarrow \gamma \gamma$ at one loop [75]. Recent advances in the generalization of the formalism have been published in Refs. [76,77].

Since scattering amplitudes are determined by their analytic properties, general considerations for integrand-level expansions should start with the propagators as the origin of divergences. The numerator, while playing a role in the appearance of UV divergences, is not relevant for the discussion of asymptotic expansions since within LTD the singular UV behavior is neutralized through local renormalization before integration.

We can reparametrize the dual propagators in the following form that is more suitable for asymptotic expansions

$$
\tilde{\delta}\left(q_{i}\right) G_{D}\left(q_{i} ; q_{j}\right)=\frac{\tilde{\delta}\left(q_{i}\right)}{2 q_{i} \cdot k_{j i}+\Gamma_{i j}+\Delta_{i j}-\imath 0 \eta \cdot k_{j i}},
$$


where $\Gamma_{i j}+\Delta_{i j}=k_{j i}^{2}+m_{i}^{2}-m_{j}^{2}$. If $\Gamma_{i j}+\Delta_{i j}$ vanishes, the dual propagator is not expanded. Otherwise, the starting point for the asymptotic expansion is to demand that the condition

$$
\left|\Delta_{i j}\right| \ll\left|2 q_{i} \cdot k_{j i}+\Gamma_{i j}\right|
$$

be fulfilled for the whole range of the loop integration space except for potentially small regions around physical divergences. Please note that since dual propagators only appear in integrands where one-loop momentum has been set on-shell, the condition must be fulfilled in the Euclidean space of the loop-three momentum. The dual propagator can then be expanded as

$$
G_{D}\left(q_{i} ; q_{j}\right)=\sum_{n=0}^{\infty} \frac{\left(-\Delta_{i j}\right)^{n}}{\left(2 q_{i} \cdot k_{j i}+\Gamma_{i j}-i 0 \eta \cdot k_{j i}\right)^{n+1}} .
$$

Further simplifications arise whenever $\mathbf{k}_{j i}=0$. (The bold denotes here the space component of the corresponding four-vector.) In that case, with the change of variables $\left|\vec{q}_{i}\right|=m_{i} / 2\left(x_{i}-x_{i}^{-1}\right)$, the denominator of the expanded dual propagator takes the easily integrable form

$$
2 q_{i} \cdot k_{j i}+\Gamma_{i j}-\imath 0 \eta \cdot k_{j i}=Q_{i}^{2}\left(x_{i}+r_{i j}\right)\left(x_{i}^{-1}+r_{i j}\right) .
$$

The intention to rewrite the denominator in this shape determines the parameters $\Gamma_{i j}$ and $r_{i j}$ appearing in the expansion to be restricted by the conditions

$$
\begin{aligned}
& \Gamma_{i j}-\imath 0 \eta \cdot k_{j i}=Q_{i}^{2}\left(1+r_{i j}^{2}\right), \\
& r_{i j}=\frac{m_{i} k_{j i, 0}}{Q_{i}^{2}}-\frac{\imath 0 \eta \cdot k_{j i}}{Q_{i}^{2}},
\end{aligned}
$$

assuming $\left|r_{i j}\right| \leq 1$. In the types of limits where one hard scale $Q$ is available, $Q_{i}^{2}= \pm Q^{2}$ can be identified. The sign is determined by the sign of the hard scale in the expression $k_{j i}^{2}+m_{i}^{2}-m_{j}^{2}$. This type of expansion facilitates the analytical integration based on integrals of the form

$$
\int_{1}^{\infty} \frac{\mathrm{d} x_{i}}{x_{i}\left(x_{i}+r_{i j}\right)\left(x_{i}^{-1}+r_{i j}\right)}=\frac{\log \left(r_{i j}\right)}{r_{i j}^{2}-1}, \quad\left|r_{i j}\right|<1 .
$$

In addition to the relations in Equation (30) further conditions must be respected by the expansion parameters to ensure that the expansion converges both at integrand and at integral level. Specifically, it is fundamental that the analytic behavior of the dual propagator is not fundamentally changed, i.e., that for a propagator with a singularity the expansion also must display that singularity, while the expansion of a non-singular propagator is to be finite throughout the whole integration domain. The infinitesimal imaginary prescription of $r_{i j}$ given in Equation (30) accounts properly for the complex prescription of the original dual propagator and therefore its causal thresholds. A different approach must be taken for threshold limits where a hard scale is not identifiable. In this case, the pole position of the non-expanded propagator can be expanded to identify the correct $r_{i j}$ for the asymptotic expansion.

The formalism of expanding the dual propagator has been developed through its application on the locally renormalized scalar two-point function

$$
\mathcal{A}^{(1, R)}=\int_{\ell}\left[G_{F}\left(q_{1} ; M\right) G_{F}\left(q_{2} ; m\right)-\left(G_{F}\left(\ell ; \mu_{\mathrm{UV}}\right)\right)^{2}\right] .
$$

Applying Equation (28) on the appearing dual propagators $G_{D}\left(q_{1} ; \ell\right)$ and $G_{D}\left(\ell ; q_{1}\right)$ leads to a simplified expression that can be integrated without needing to specify what type of limit is considered, giving the very general result 


$$
\mathcal{A}^{(1, R)}=\frac{1}{16 \pi^{2}} \sum_{i, j}\left[2+c_{0, i} \log \left(\frac{\mu_{\mathrm{UV}}}{m_{i}}\right)+\sum_{n=0}^{\infty}\left(c_{1, i}^{(n)}+c_{2, i}^{(n)} \log \left(r_{i j}\right)\right)\right] .
$$

The coefficients $c$ are simple functions of the appearing scales and are given in Ref. [77] just as with the parameters needed for different limits. The expansion converges well both at integrand- and at integral level in the limits of one large mass, a large external momentum as well as when approaching the physical threshold both from below and from above. Comparison with the established method of expansion by regions [104-108] has shown faster convergence as well as emphasized the advantage of decreasing degree in UV divergence with each order in the expansion. Renormalization within our method thus only involves the lowest orders of the integrand-level expansion. Higher terms are optional for increasing precision and can be added straight-forwardly without the need to ensure further UV cancellation.

Using the same approach, we have found integrand level expansions for the scalar three-point function

$$
\mathcal{A}_{3}^{(1)}=\int_{\ell} G_{F}\left(q_{1}, q_{2}, q_{3} ; M\right),
$$

both for the limit of a large mass

$$
\mathcal{A}_{3}^{(1)}\left(s_{12} \ll M^{2}\right)=-\frac{1}{16 \pi^{2}} \frac{1}{2 M^{2}}\left(1+\frac{r}{12}+\frac{r^{2}}{90}\right)+\mathcal{O}\left(r^{3}\right),
$$

as well as for a small mass

$$
\mathcal{A}_{3}^{(1)}\left(s_{12} \gg M^{2}\right)=\int_{\ell} \frac{\tilde{\delta}(\ell ; M) s_{12}}{\left(2 \ell \cdot p_{12}\right)\left(2 \ell \cdot p_{1}\right)} \sum_{n=0}^{\infty}\left\{\frac{M^{2 n}}{\left(-2 \ell \cdot p_{12}+\Gamma\right)^{n+1}}+\frac{M^{2 n}}{\left(2 \ell \cdot p_{12}+\Gamma\right)^{n+1}}\right\} \text {. }
$$

The Euclidean structure of the dual integrand, $a(\ell)$, can be exploited into an even more direct way by applying Taylor expansions. Here it is important to use different assumptions on the size of the loop-three momentum in separate parts of the integration range. For the scalar two-point function in the large-mass limit this amounts to calculating

$$
\mathcal{A}=\int_{0}^{\lambda} \mathrm{d}|\ell| \mathcal{T} a(M, \infty)+\int_{\lambda}^{\infty} \mathrm{d}|\ell| \mathcal{T} a(\{\ell, M\}, \infty) .
$$

The expanded integrand converges well in the full range of the loop momentum as can be seen in Figure 7. Details on this method and on how to fix the matching scale lambda that separates the appearing so-called dual regions can be found in [77].

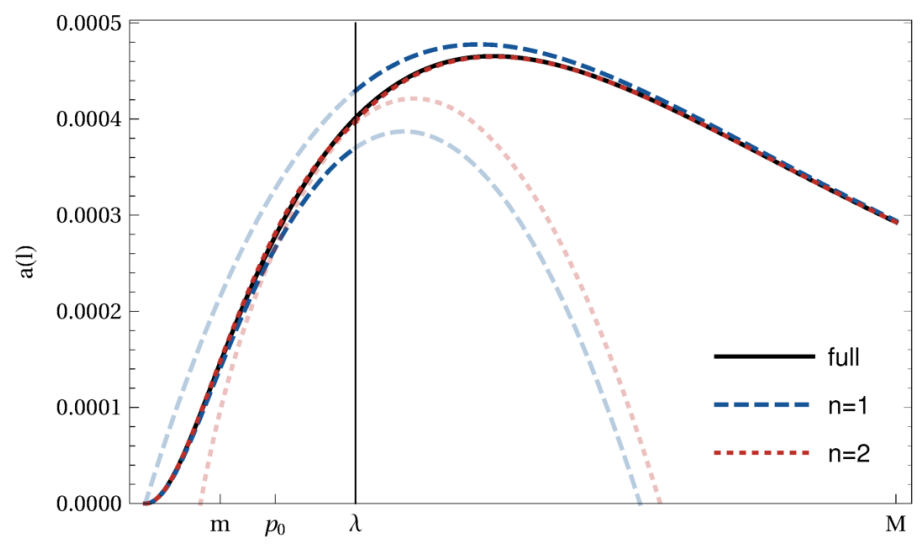

$|\vec{l}|$

Figure 7. The convergence at integrand level of the expansion given in Equation (38) for the values $M=10 \mathrm{~m}, p^{2}=3 \mathrm{~m}^{2}$, and $\mu_{\mathrm{UV}}=M$. More details about this plot can be found in the original paper [77]. 
With the calculations above being performed in the traditional LTD representation we will finally show an example of using the manifestly causal representation to expand a multi-loop integral. The MLT structure offers an ideal starting point, being void of unphysical singularities. For the large-mass limit, we find

$$
\mathcal{A}_{\mathrm{MLT}}^{(L)}\left(p^{2} \ll m_{s}^{2}\right)=-2 \sum_{n=0}^{\infty}\left(p^{2}\right)^{n} \int_{\vec{\ell}_{1}, \ldots, \vec{\ell}_{L}} \frac{\left(\lambda_{L+1}^{0}\right)^{-1-2 n}}{x_{L+1}},
$$

where $\lambda_{L+1}^{0}=\sum_{s=1}^{L+1} q_{s, 0}^{(+)}$and $x_{L+1}=\prod_{s=1}^{L+1} 2 q_{s, 0}^{(+)}$. Notice that due to the lack of dependence on $p_{0}$ both in $x_{L+1}$ nor in $\lambda_{L+1}^{0}$ the asymptotic integrals on the right-hand side of Equation (39) are a function only of the internal masses, $m_{s}$, to all-loop orders. In this way, we are optimistic about future applications of the manifestly causal LTD representation from Refs. [92,95-97] to speed up the calculation of efficient and smooth asymptotic expansions.

\section{Manifestly Causal Representation and Numerical Efficiency}

As explained in Sections 2 and 3, the application of the nested residue strategy leads to manifestly causal integrand-level representations of any multi-loop multi-leg Feynman amplitude. The main advantage of such a representation is the absence of non-physical singularities, because only terms compatible with causality remain in the final result.

In the following Section, we show the explicit causal formulae for NMLT and $\mathrm{N}^{2} \mathrm{MLT}$ families for an arbitrary number of loops, and discuss their interpretation in terms of entangled causal thresholds. Numerical examples for 4-loop diagrams are presented, to provide a comparison with available results and test the efficiency of our approach. It is worth appreciating that these compact formulae were obtained using the package FINITEFLOW [109], which implements an algorithm for the numerical evaluation over finite fields $[47,110]$, and allows us to explicitly reconstruct the causal representation.

\subsection{Next-to-Maximal Loop Topology (NMLT)}

At three loops, the MLT family is not enough to describe the whole set of possible topologies. Thus, we need to consider the NMLT and $\mathrm{N}^{2}$ MLT topologies, whose general dual representations were explained in Section 2. To simplify the presentation, we start by considering NMLT configurations with one single propagator in each set and no external momenta. We need to add an additional internal line, whose momentum is given by

$$
q_{L+2}=-\ell_{1}-\ell_{2}
$$

A pictorial representation of NMLT is provided in Figure 1b. After computing the nested residues and adding all the contributions together, we obtain

$$
\mathcal{A}_{\mathrm{NMLT}}^{(L)}(1,2, \ldots, L+2)=\int_{\vec{\ell}_{1}, \ldots, \vec{\ell}_{L}} \frac{2}{x_{L+2}}\left(\frac{1}{\lambda_{1} \lambda_{2}}+\frac{1}{\lambda_{2} \lambda_{3}}+\frac{1}{\lambda_{3} \lambda_{1}}\right),
$$

where the causal propagators are given by

$$
\lambda_{1}=\sum_{i=1}^{L+1} q_{i, 0}^{(+)} \quad \lambda_{2}=q_{1,0}^{(+)}+q_{2,0}^{(+)}+q_{L+2,0}^{(+)} \quad \lambda_{3}=\sum_{i=3}^{L+2} q_{i, 0}^{(+)} .
$$

This expression was reconstructed using numerical evaluations over finite fields, although partial fractioning leads to the same result within a similar computing time. In the following, we shall note that simplifications are not straightforward when dealing with more complicated topologies.

An interesting consequence of the compact form of Equation (41) is that it allows a reinterpretation in in terms of entangled causal thresholds. Each $\lambda_{i}$ is associated with a causal threshold singularity, which might take place when the momenta flows are oriented in the same direction. Thus, the product of causal denominators represents a configuration in 
which two (or more) sets of propagators can simultaneously go on-shell. The factorization of NMLT (and more complicated topologies) as products of MLT configurations is the reason behind this behavior [92]. A graphical interpretation of the entangled causal structure of NMLT vacuum diagrams is provided in Figure 8, with the dashed lines indicating the propagators that can be set simultaneously on-shell.

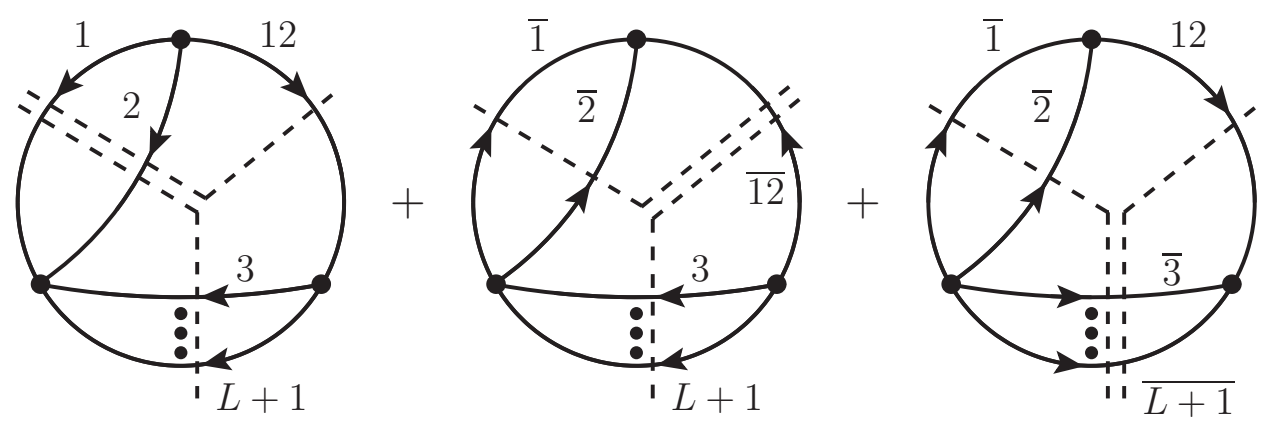

Figure 8. Entangled causal thresholds of the NMLT topology. Products of two causal propagators are involved.

\subsection{Next-to-Next-to-Maximal Topology ( $\left.N^{2} M L T\right)$}

As mentioned before, reaching a full description of $\mathrm{N}^{k}$ MLT with $k \leq 2$ is enough to obtain the causal representation of up to three-loop scattering amplitudes. The socalled $\mathrm{N}^{2}$ MLT can be built recursively from the NMLT by adding an additional line with momentum

$$
q_{L+3}=-\ell_{2}-\ell_{3}
$$

The minimal example of such topology is the Mercedes-Benz diagram $(L=3)$ shown in Figure 1c. For the sake of simplicity, we restrict here to the case without external momenta. Using the LTD representation in Ref. [92], we can add together all the contributions and obtain

$$
\mathcal{A}_{\mathrm{N}^{2} \mathrm{MLT}}^{(L)}(1,2, \ldots, L+3)=\int_{\vec{\ell}_{1}, \ldots, \vec{\ell}_{L}} \frac{1}{x_{L+3}} \frac{\mathcal{N}\left(\left\{q_{i, 0}^{(+)}\right\}\right)}{\prod_{i=1}^{7} \lambda_{i}},
$$

with $\lambda_{1}$ through $\lambda_{3}$ defined in Equation (42),

$$
\begin{array}{ll}
\lambda_{4}=q_{2,0}^{(+)}+q_{3,0}^{(+)}+q_{L+3,0}^{(+)}, & \lambda_{6}=q_{1,0}^{(+)}+q_{3,0}^{(+)}+q_{L+2,0}^{(+)}+q_{L+3,0}^{(+)}, \\
\lambda_{5}=q_{1,0}^{(+)}+q_{L+3,0}^{(+)}+\sum_{i=4}^{L+1} q_{i, 0}^{(+)}, & \lambda_{7}=q_{2,0}^{(+)}+\sum_{i=4}^{L+3} q_{i, 0}^{(+)},
\end{array}
$$

and $\mathcal{N}\left(\left\{q_{i, 0}^{(+)}\right\}\right)$a degree-four polynomial in $q_{i, 0}^{(+)}$. Compared to the NMLT case, the complexity of the polynomial in the denominator makes it highly non-trivial to unveil a formula similar to Equation (41). Thus, we rely on the analytic reconstruction over finite fields to obtain

$$
\begin{aligned}
\mathcal{A}_{\mathrm{N}^{2} \mathrm{MLT}}^{(L)}(1,2, \ldots, L+3)= & -\int_{\vec{\ell}_{1}, \ldots, \vec{\ell}_{L}} \frac{2}{x_{L+3}}\left[\frac{1}{\lambda_{1}}\left(\frac{1}{\lambda_{2}}+\frac{1}{\lambda_{3}}\right)\left(\frac{1}{\lambda_{4}}+\frac{1}{\lambda_{5}}\right)\right. \\
& \left.+\frac{1}{\lambda_{6}}\left(\frac{1}{\lambda_{2}}+\frac{1}{\lambda_{4}}\right)\left(\frac{1}{\lambda_{3}}+\frac{1}{\lambda_{5}}\right)+\frac{1}{\lambda_{7}}\left(\frac{1}{\lambda_{2}}+\frac{1}{\lambda_{5}}\right)\left(\frac{1}{\lambda_{3}}+\frac{1}{\lambda_{4}}\right)\right],
\end{aligned}
$$

whose graphical representation in terms of entangled thresholds is given in Figure 9.

It is worth appreciating that the package LOTTY was used to efficiently reach these results [101]. As in the case of NMLT topologies, it is possible to interpret this result using entangled thresholds. This time, there are products of three causal propagators. Notice that not all the combinations of causal propagators are allowed. This is because causal 
propagators exhibit some compatibility issues, which can be explained by digging into graph theory. More details about this issue and the connection with Cutkosky's rules can be found in Ref. [102].
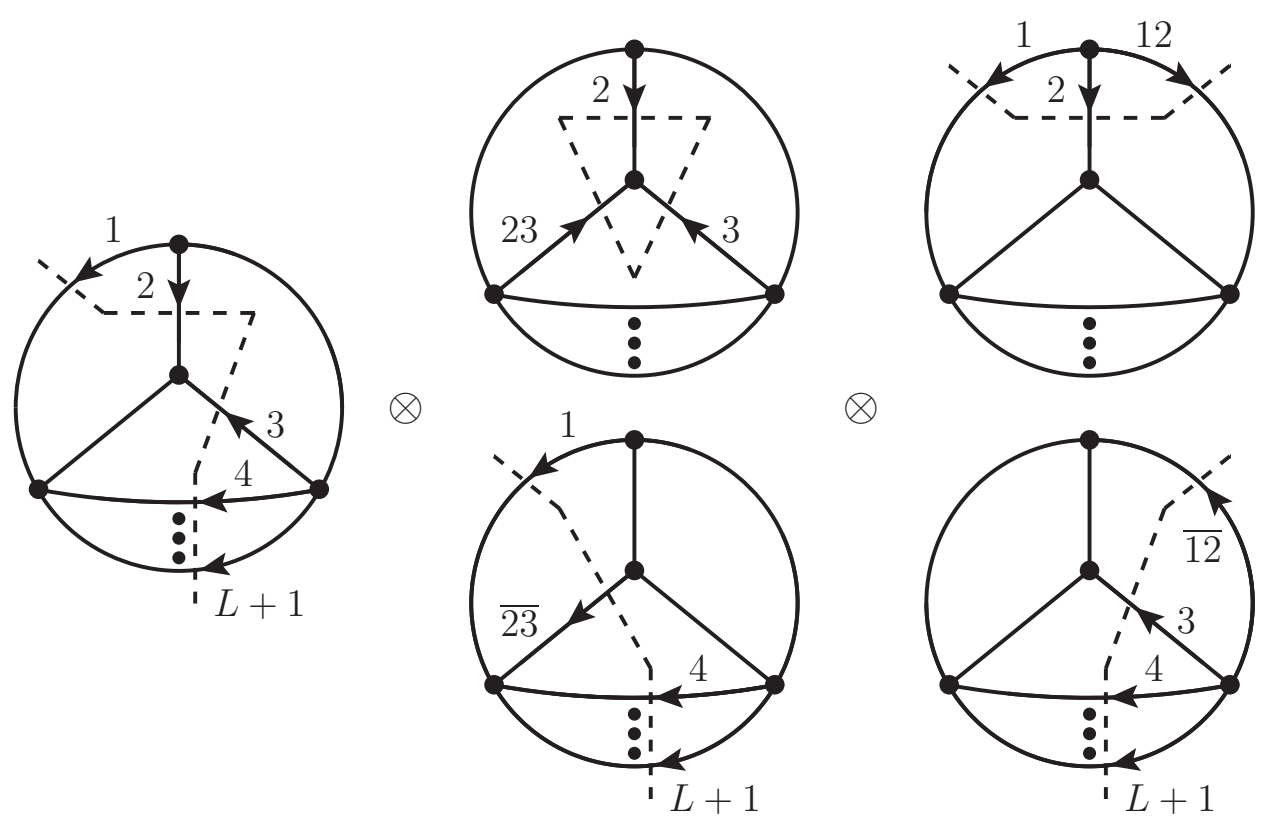

$\bigotimes$

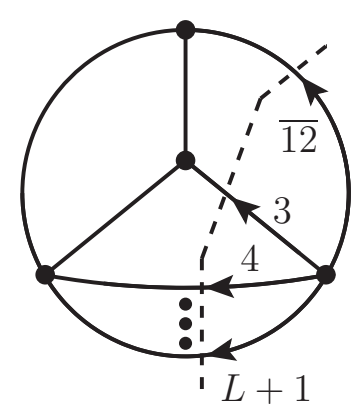

Figure 9. Entangled causal thresholds of the $\mathrm{N}^{2} \mathrm{MLT}$ topology. Products of three causal propagators are involved.

\subsection{Adding External Momenta and Higher-Powers}

For realistic multi-loop scattering amplitudes, we need to take into account external legs. The analytic reconstruction algorithm used to obtain compact formulae for vacuum diagrams can be also applied to topologies with external momenta. We want to highlight that the insertion of external momenta does not affect the causal physical behavior of these integrals: the difference regarding the vacuum case is that the entangled configurations are duplicated, according to the direction of the energy flow for external particles. For instance, a generic $\mathrm{N}^{2} \mathrm{MLT}$ with external legs inserted in the vertices is given by

$$
\begin{aligned}
\mathcal{A}_{\mathrm{N}^{2} \mathrm{MLT}}^{(L)}\left(1,2, \ldots,(L+1)_{-p_{13^{\prime}}}(L+2)_{p_{2^{\prime}}}(L+3)_{-p_{3}}\right)=-\int_{\vec{l}_{1}, \cdots, \vec{\ell}_{L}} \frac{1}{x_{L+3}} \\
\times\left[\frac{1}{\lambda_{1}^{+}}\left(\frac{1}{\lambda_{2}^{-}}+\frac{1}{\lambda_{3}^{-}}\right)\left(\frac{1}{\lambda_{4}^{+}}+\frac{1}{\lambda_{5}^{+}}\right)+\frac{1}{\lambda_{6}^{+}}\left(\frac{1}{\lambda_{3}^{-}}+\frac{1}{\lambda_{5}^{-}}\right)\left(\frac{1}{\lambda_{2}^{+}}+\frac{1}{\lambda_{4}^{+}}\right)\right. \\
\left.\quad+\frac{1}{\lambda_{7}^{+}}\left(\frac{1}{\lambda_{3}^{-}}+\frac{1}{\lambda_{4}^{-}}\right)\left(\frac{1}{\lambda_{2}^{+}}+\frac{1}{\lambda_{5}^{+}}\right)+\left(\lambda_{i}^{+} \leftrightarrow \lambda_{i}^{-}\right)\right] .
\end{aligned}
$$

More details about the algorithms used to perform these computations can be found in Refs. [96,101].

Besides that, it was shown in Refs. $[82,84,85,111]$ that the presence of self-energy insertions or generic scattering amplitudes, as well as some local UV counter-terms, might require consideration of propagators with higher-powers. As explained in Refs. [96,97], the causal structure of these amplitudes can be obtained by applying a differential operator. Explicitly, we can raise the power of the propagators by taking derivatives regarding $q_{i, 0}^{(+)}$. For instance,

$$
\left(G_{F}\left(q_{i}\right)\right)^{\alpha_{i}}=\frac{1}{\left(\alpha_{i}-1\right) !} \frac{\partial^{\alpha_{i}-1}}{\partial\left(\left(q_{i, 0}^{(+)}\right)^{2}\right)^{\alpha_{i}-1}} G_{F}\left(q_{i}\right),
$$


which suggests the definition of the operator

$$
\frac{\partial}{\partial\left(q_{i, 0}^{(+)}\right)^{2}} \bullet=\frac{1}{2 q_{i, 0}^{(+)}} \frac{\partial}{\partial\left(q_{i, 0}^{(+)}\right)} \bullet
$$

The iterated application of this operator to the causal representation of scattering amplitudes with single powers of the denominators leads to causal representation of the corresponding multi-power amplitude, as carefully explained in Ref. [97].

\subsection{Numerical Implementations}

Finally, we would like to highlight that the causal representation of multi-loop multileg Feynman integrals and scattering amplitudes leads to a very smooth numerical integration. In Ref. [96], we studied several examples and compared the performance of the LTD-inspired approach with other standard frameworks (such as FIESTA 4.2 and SECDEC 3.0). We considered the generic scalar amplitude

$$
\begin{aligned}
\mathcal{A}_{\mathrm{N}^{k-1} \mathrm{MLT}}^{(L)}\left(1^{2}, 2^{2}, \ldots, L^{2}\right. & , L+1, \ldots, L+k) \\
& =\prod_{i=1}^{L} \frac{\partial}{\partial\left(q_{i, 0}^{(+)}\right)^{2}} \mathcal{A}_{\mathrm{N}^{k-1} \mathrm{MLT}}^{(L)}(1,2, \ldots, L+1, \ldots, L+k),
\end{aligned}
$$

with $k=\{0,1,2\}$, at three and four loops, and changing the dimensionality of the space-time from $d=2$ to $d=4$. Here, we applied the differential operator detailed in Section 5.3 to raise the powers of the denominators and achieve integrability in the UV region. In Figures 10-12, we show a few examples of Equation (50) for $d=4$, doing a scan in $m_{4}^{2}$, by fixing $m_{5}^{2}$.

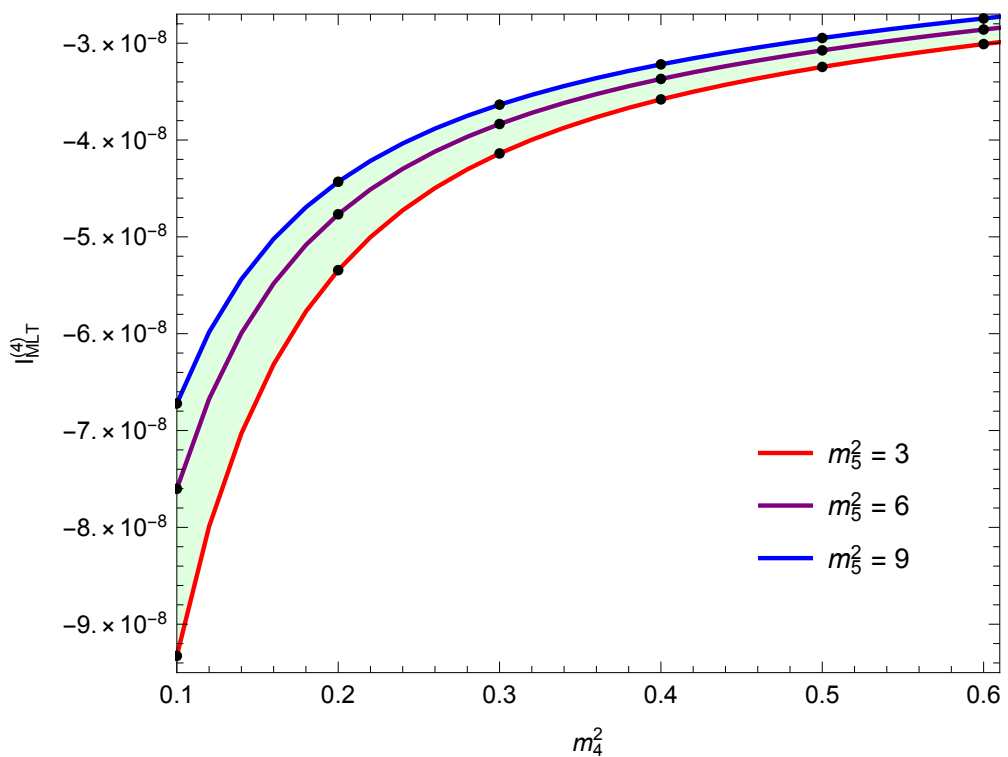

Figure 10. Four-dimensional MLT at four loops, as a function of the internal masses $m_{4}^{2}$ and $m_{5}^{2}$. Although the solid lines correspond to the results obtained within the LTD formalism, the dots are the numerical results obtained with FIESTA 4.2. 


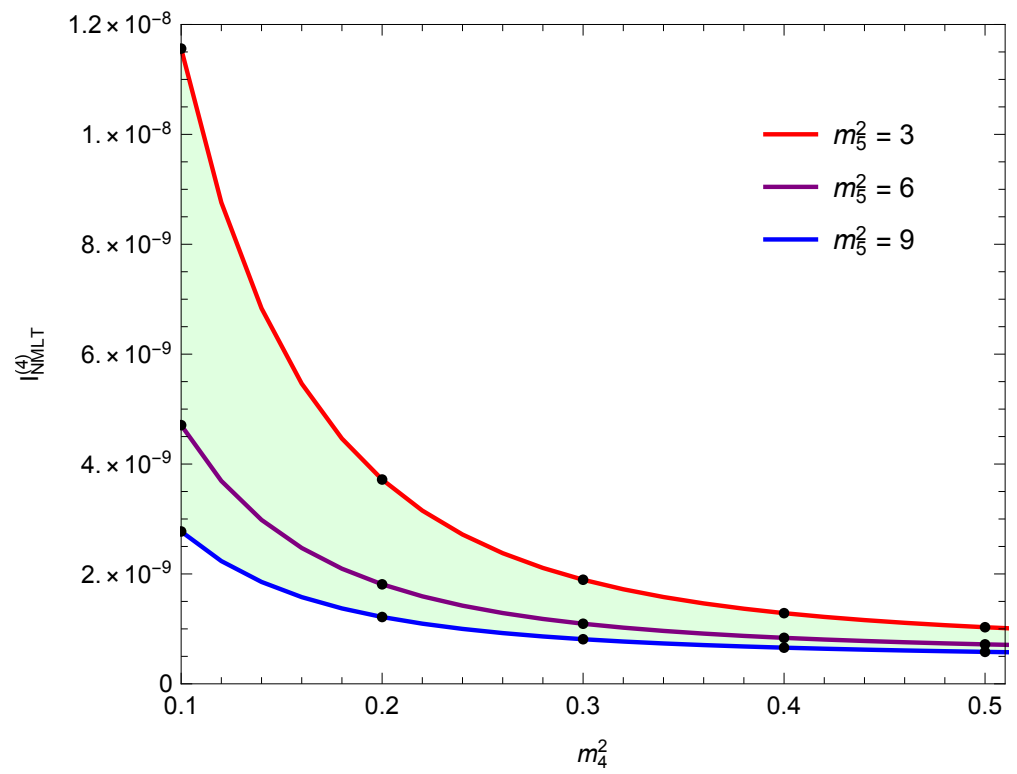

Figure 11. Four-dimensional NMLT at four loops, as a function of the internal masses $m_{4}^{2}$ and $m_{5}^{2}$. Although the solid lines correspond to the results obtained within the LTD formalism, the dots are the numerical results obtained with FIESTA 4.2.

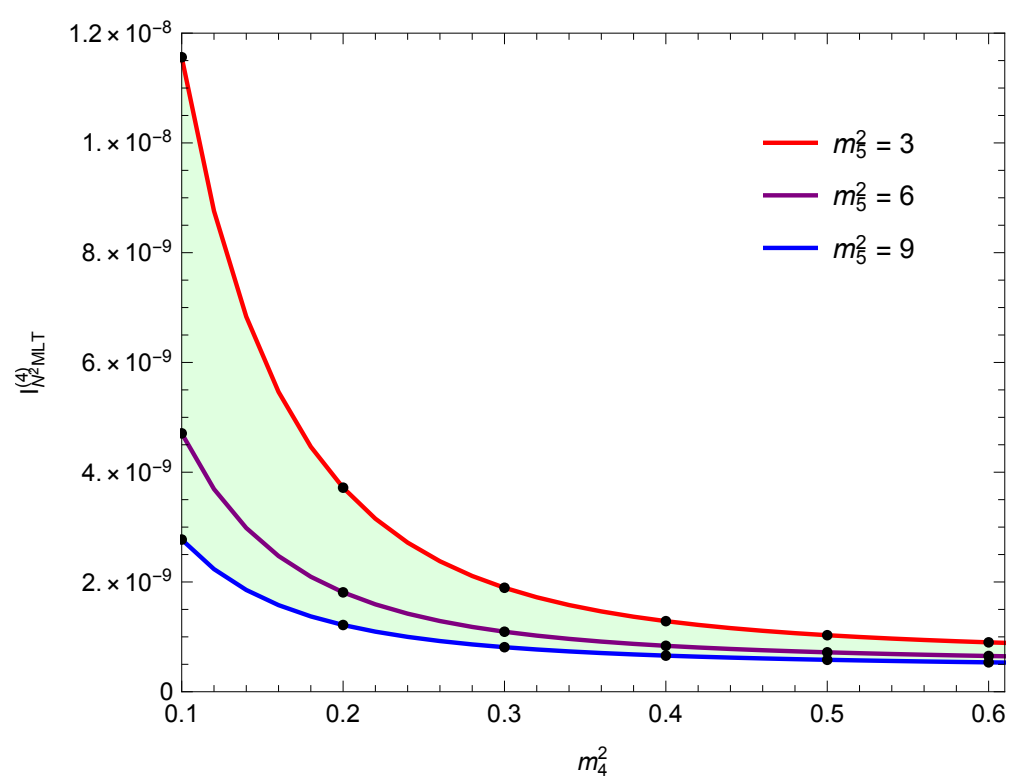

Figure 12. Four-dimensional $\mathrm{N}^{2} \mathrm{MLT}$ at four loops, as a function of the internal masses $m_{4}^{2}$ and $m_{5}^{2}$. Although the solid lines correspond to the results obtained within the LTD formalism, the dots are the numerical results obtained with FIESTA 4.2.

The inclusion of arbitrary masses does not introduce any additional complication within the LTD-based framework. Additionally, only $(d-1)$ integrations for each loop are needed, while any methodology based on the traditional Feynman parametrization approaches scales with the number of propagators. Moreover, we explicitly checked that the absence of noncausal singularities leads to a very efficient integration. The technology to perform these calculations was included in the WOLFRAM MATHEMATICA package LOTTY, recently published by one of the authors [101].

\section{Universal Opening at Four Loops}

The following multi-loop topologies in complexity are those emerging at four loops: the next-to-next-to-next-to maximal loop topology $\left(\mathrm{N}^{3} \mathrm{MLT}\right)$ and next-to-next-to-next-to- 
next-to maximal loop topology ( $\left.\mathrm{N}^{4} \mathrm{MLT}\right)$ which are represented by multi-loop diagrams with $L+4$ and $L+5$ sets of propagators, respectively. In fact, $\mathrm{N}^{4} \mathrm{MLT}$ naturally includes all $\mathrm{N}^{k-1}$ MLT configurations, with $k \leq 4$.

The $\mathrm{N}^{4}$ MLT family is represented by three topologies. These topologies can be associated with the fitting of a four-point subamplitude, therefore, the fused is set in what will be known as universal topology. Each of the individual topologies are construed as the $t-, \mathrm{s}-$ and $u$-kinematic channels and are shown in Figure 13. Is important to mention that the selection is validated using QGRAF [112].
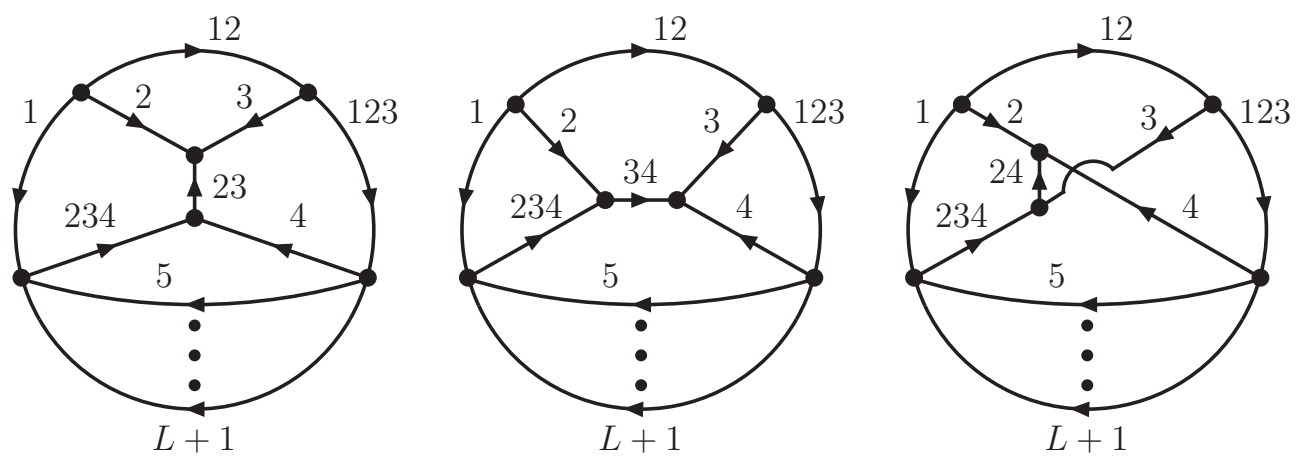

Figure 13. Diagrams of the $\mathrm{N}^{4}$ MLT family. The diagram on the left-hand side corresponds to the $t$ channel, the diagram on the center is the $s$ channel and the diagram on the right-hand side corresponds to the $u$ channel. External particles are not shown.

From the total $L+5$ sets of propagators contained in the three topologies, $L+4$ are common sets and one is distinct for each of them. The first $L$ sets of propagators only depend on a single loop momentum $\ell_{\mathfrak{S}}$, the subsequent common sets are settled as linear combinations of the loop momenta. The additional sets are the key to distinguish the three channels in the universal topology where the momenta of their propagators is identified as different linear combinations of $\ell_{2}, \ell_{3}$ and $\ell_{4}$.

To assemble the three $\mathrm{N}^{4} \mathrm{MLT}$ channels into a single topology, a current $J$ is defined as the union of three sets that characterize each channel,

$$
J \equiv 23 \cup 34 \cup 24 .
$$

Given the constraint imposed by momentum conservation, the three subsets do not act trough the same independent Feynman diagram but all of them contribute at amplitude level. According on the framework presented above, the $\mathrm{N}^{4} \mathrm{MLT}$ universal topology Feynman representation is written as

$$
\mathcal{A}_{\mathrm{N}^{4} \mathrm{MLT}}^{(L)}(1, \ldots, L+1,12,123,234, J)=\int_{\ell_{1}, \ldots, \ell_{L}} \mathcal{A}_{F}^{(L)}(1, \ldots, L+1,12,123,234, J) .
$$

The dual opening of this topology fulfills a factorization identity similar to those presented in Ref. [92] for NMLT and $\mathrm{N}^{2}$ MLT

$$
\begin{aligned}
\mathcal{A}_{\mathrm{N}^{4} \mathrm{MLT}}^{(L)} & (1, \ldots, L+1,12,123,234, J) \\
& =\mathcal{A}_{\mathrm{N}^{4} \mathrm{MLT}}^{(4)}(1,2,3,4,12,123,234, J) \otimes \mathcal{A}_{\mathrm{MLT}}^{(L-4)}(5, \ldots, L+1) \\
& +\mathcal{A}_{\mathrm{N}^{2} \mathrm{MLT}}^{(3)}(1 \cup 234,2,3,4 \cup 123,12, J) \otimes \mathcal{A}_{\mathrm{MLT}}^{(L-3)}(\overline{5}, \ldots, \overline{L+1}),
\end{aligned}
$$

also valid despite the internal configuration. The diagrammatic representation of this factorization identity is shown in Figure 14 and is called the universal identity given the fact that it is the only expression needed to open any scattering amplitude of up to four loops to nondisjoint trees. Furthermore, it allows the causal behavior of the complete topology to be inferred by looking at the causal structure of its subtopologies. 

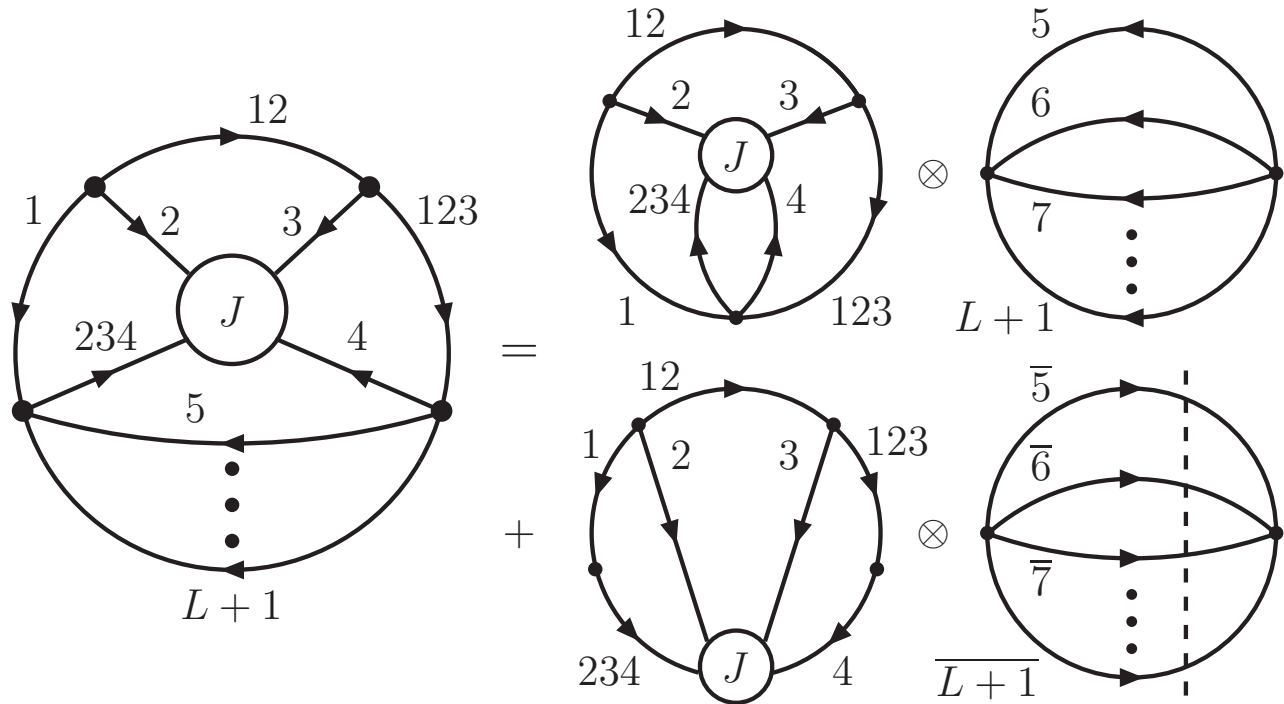

Figure 14. Diagrammatic representation for the factorized opening of the multi-loop $\mathrm{N}^{4} \mathrm{MLT}$ universal topology. Only the on-shell cut of the last MLT-like subtopology with reversed momentum flow is shown.

The term $\mathcal{A}_{\mathrm{N}^{4} \mathrm{MLT}}^{(4)}$ on the right-hand side of Equation (53) considers all possible configurations with four on-shell propagators in the sets $\{1,2,3,4,12,123,234, J\}$, while $\mathcal{A}_{\mathrm{N} 2}^{(3)} \mathrm{MLT}$ in the second term assumes three on-shell conditions. The terms $\mathcal{A}_{\mathrm{MLT}}^{(L-4)}(5, \ldots, L+1)$ and $\mathcal{A}_{\mathrm{MLT}}^{(L-3)}(\overline{5}, \ldots, \overline{L+1})$ are computed according to Ref. $[92,97]$.

The four-loop subtopology in Equation (53) is opened through a factorization identity which is written in terms of known subtopologies,

$$
\begin{aligned}
\mathcal{A}_{\mathrm{N}^{4} \mathrm{MLT}}^{(4)}(1,2,3,4,12,123,234, J) & =\mathcal{A}_{\mathrm{N}^{2} \mathrm{MLT}}^{(4)}(1,2,3,4,12,123,234) \otimes \mathcal{A}^{(0)}(J) \\
& +\sum_{\mathbf{s} \in J} \mathcal{A}_{D}^{(4)}(1,2,3,4,12,123,234, \mathbf{s}) .
\end{aligned}
$$

Exhibiting a similar structure to Equation (54), the three-loop subtopology in Equation (53) is given by

$$
\begin{aligned}
\mathcal{A}_{\mathrm{N}^{2} \mathrm{MLT}}^{(3)}(1 \cup 234,2,3,4 \cup 123,12, J) & =\mathcal{A}_{\mathrm{NMLT}}^{(3)}(1 \cup 234,2,3,4 \cup 123,12) \otimes \mathcal{A}^{(0)}(J) \\
& +\sum_{\mathbf{s} \in J} \mathcal{A}_{D}^{(3)}(1,2,3,4,12,123,234, \mathbf{s}),
\end{aligned}
$$

where the bold symbol $\mathbf{s}$ is used to indicate that these contributions are those containing on-shell propagators in the $J$-sets.

The first term on the right-hand side of Equations (54) and (55) consists of a four-loop $\mathrm{N}^{2} \mathrm{MLT}$ and three-loop NMLT subtopology, respectively. These terms are describing dual trees where all the propagators with momenta in $J$ remain off-shell. The second term on the right-hand side of Equations (54) and (55) collects contributions that characterize the $s$, $t$ or $u$ channel shown in Figure 13. To obtain these contributions a propagator in either set 23,34 or 24 are set on-shell.

The explicit expressions for the $s, t$ and $u$ channel arising from Equations (54) and (55) are presented in Ref. [95] where all the outcomes are in accordance with the absence of disjoint trees. Notice that the number of dual terms in the LTD representation can also be obtained through a combinatorial exercise of selecting those terms that do not generate disjoint trees; however, the application of the nested residues is the only way to display the momentum flows of the on-shell propagators. Moreover, it is relevant to be aware that the number of elements for any $\mathrm{N}^{k-1}$ MLT topology in Equation (53) scales with the number 
of loops and propagators per loop set, linearly. On the other hand, the sum over residues over internal propagators, is implicitly taken into account in this expression.

\section{Causal Representations}

To confirm the causal conjecture for the $\mathrm{N}^{4} \mathrm{MLT}$ family, the strategy proposed in Ref. [96] is applied to the multi-loop $\mathrm{N}^{3} \mathrm{MLT}, t, s$ and $u$ channels. Each topology considers a configuration with one internal propagator in each loop set, four external momenta for $\mathrm{N}^{3} \mathrm{MLT}$ and six external particles for $t, s$ and $u$ channels.

As a first step, the LTD representation is obtained for each of the selected topologies through the universal $\mathrm{N}^{4} \mathrm{MLT}$ expression in Equation (53). After computing the nested residues and adding them all together a causal expression is found. The integrand of the causal dual representation reads in terms of on-shell energies, the energy components of the linear combination and causal denominators. Let us remember and emphasize that these causal denominators are constructed from sums of on-shell energies exclusively, and they represent potential singular configuration.

The results given by the straightforward application of LTD leads directly to a manifestly causal expression; however, the resulting numerator is a lengthy polynomial in the on-shell and external energies [95]. Therefore, a way to obtain a more suitable causal expression is to reinterpret it in terms of entangled thresholds as defined in Ref. [96].

The analysis was performed only for scalar integrals since they consider all the required causal combinations. If there is a need to deal with tensor integrals, tensor reduction can be applied given that it commutes with LTD. Another property to benefit from is that external momenta added to interaction vertices connecting different loop sets do not modify the number of internal propagators or the complexity of the causal representation. Hence, the complete causal expressions considering external moments can be obtained from the causal representation of the vacuum configuration by aligning the momentum fluxes of the entangled thresholds.

Starting with the multi-loop $\mathrm{N}^{3} \mathrm{MLT}$, there are 13 causal denominators which are depicted in Figure 15. The analytically reconstruction was done by matching all combinations of four thresholds that are causally compatible to each other.

Going forward to the causal representation of the $\mathrm{N}^{4} \mathrm{MLT}$ family, we must consider all the entangled configurations with the presence of five causal thresholds. The $t$ channel depends on the causal denominators already defined for the $\mathrm{N}^{3}$ MLT configuration and additional nine extra causal denominators that depend on $q_{23,0}^{(+)}$where the corresponding configurations are shown in Figure 16.

The s-channel can be obtained through the structure of the $t$-channel, specifically, the causal denominators can be determined given an appropriate clockwise rotation of the $t$ channel. The $u$-channel is managed in a similar way to the $s$-channel, a proper substitution of the arguments of the causal denominators are applied in the $t$-channel: the exchange $3 \leftrightarrow 4$ or $2 \leftrightarrow 234$, remaining 123 invariant, and the substitution $23 \rightarrow 24$. However, given that the $u$-channel is non-planar three new configurations appear. These additional configurations are depicted in Figure 17. 

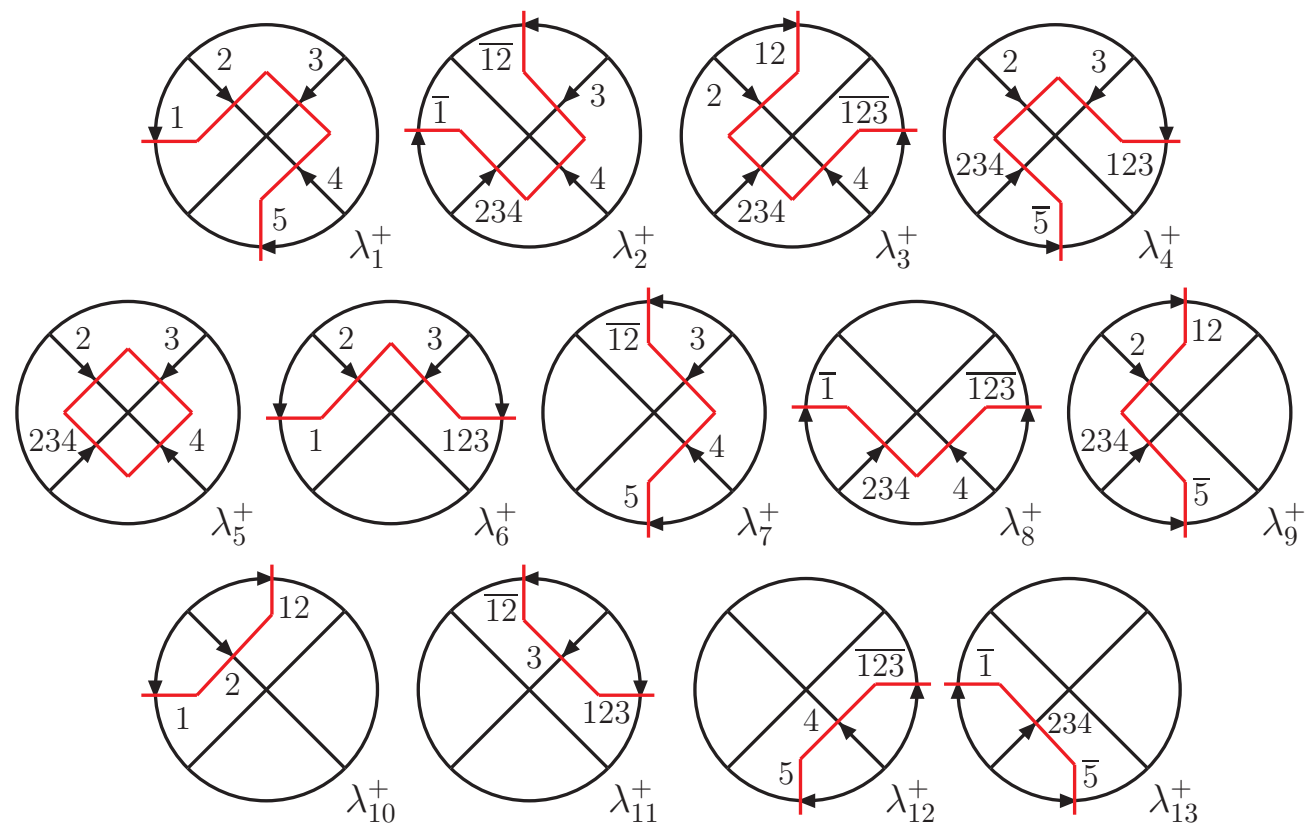

Figure 15. Causal configurations of the $N^{3}$ MLT topology. The set number 5 accounts for all the propagators in the sets 5 to $L+1$.
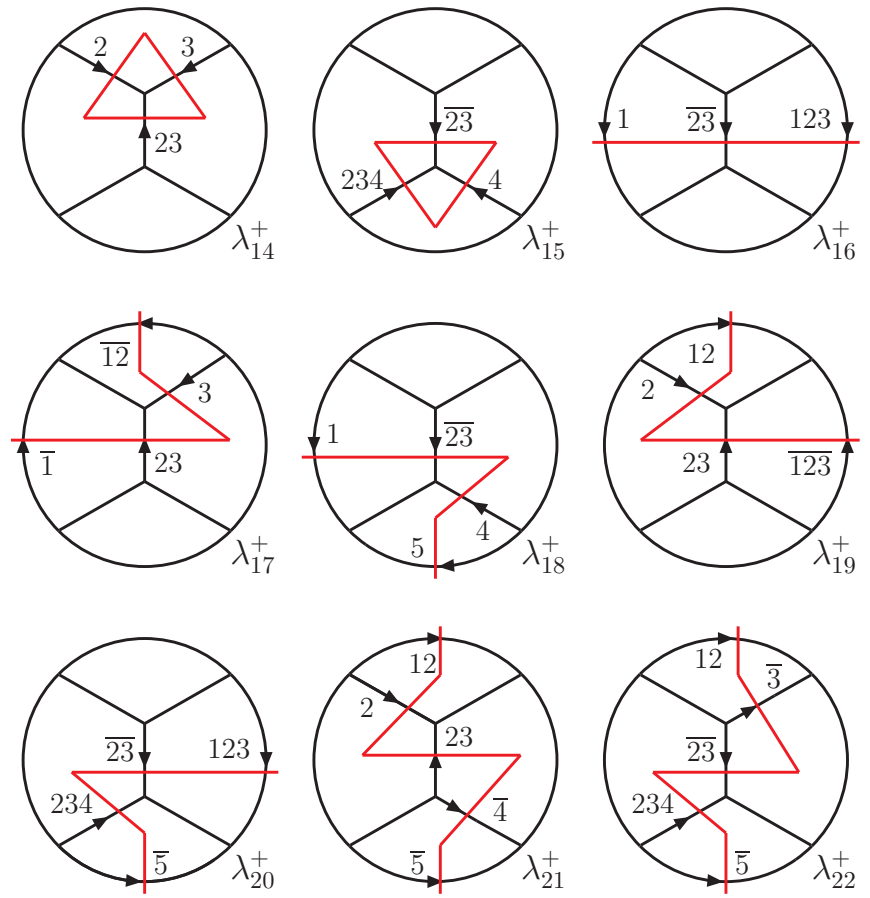

Figure 16. Extra causal configurations of the $t$-channel of the $\mathrm{N}^{4} \mathrm{MLT}$ topology.
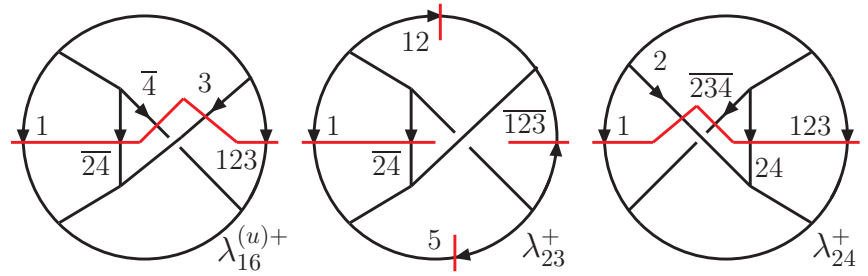

Figure 17. Extra causal configurations of the $u$-channel of the $\mathrm{N}^{4} \mathrm{MLT}$ topology due to non-planarity.

An important aspect to keep in mind is that working with the causal representation requires description of additional causal thresholds and causal entanglements when 
added internal propagators are included. Nevertheless, we have a major advantage, the number of terms for a given $\mathrm{N}^{k-1}$ MLT topology does not dependent on the number of loops considered.

Furthermore, the most significant advantage of the causal representation with respect to the LTD representation comes from the absence or presence of noncausal singularities, the main distinction between them.

The nested residue direct application leads to multiple threshold singularities, but with an ingenious analytical rearrangement we can achieve a causal representation. This representation is free of noncausal singularities and leads to a more stable and efficient numerically in the complete integration domain [95].

\section{Novel Developments on Causality}

The applications of the LTD-inspired methods have been spreading very fast in recent years. In particular, it turned out to be an excellent tool to unveil the structure of causal singularities of multi-loop multi-leg Feynman integrals and scattering amplitudes. There have been very recent findings regarding general all-loop formulae to describe any $\mathrm{N}^{k} \mathrm{MLT}$ amplitude, using clever algebraic relations among them [100]. This computational technology has been implemented in the package LOTTY [101], which allows automatic obtaining of the causal representation of multi-loop Feynman integrals and scattering amplitudes.

On the other hand, investigations inquiring on the geometrical aspects of multi-loop diagrams were performed. In Ref. [102], we used concepts from graph theory to describe all the possible causal propagators and the allowed entangled thresholds associated with a given diagram. Describing diagrams in terms of vertices (i.e., interaction vertices) and edges (i.e., sets of propagators connecting two fixed vertices) was also proposed in Ref. [100]. This description leads to the vertex matrix, a geometrical object that allows one to completely characterize the causal structure of a given diagram. Explicitly, we realized that the causal propagators are associated with all the possible connected binary partitions of vertices. This has a very nice connection to a generalized geometrical interpretation of the Cutkosky's rules [88]. Additionally, we implemented a computational algorithm that benefits from graph theory tools to efficiently obtain all the causal propagators. We tested it against the direct nested residue calculation and subsequent identification of $\lambda^{\prime}$ s: the geometric algorithm was, at least, a factor ten faster.

The information contained in the vertex matrix allow us to exactly reconstruct all the possible entangled thresholds by imposing geometrical selection criteria. These can be summarized in the following list:

1. All the lines are cut: Each possible entangled combination of causal denominators must involve the on-shell energies of all the propagators.

2. Absence of crossings: Causal denominators are associated with connected partitions of vertices. Only those partitions involving disjoint sets of vertices, or those that are strictly included, can be successfully entangled. Alternatively, if we associate a line connecting the cut edges to each $\lambda$, then two $\lambda^{\prime}$ 's can be entangled if their lines do no cross.

3. Consistent flux orientation: A set of causal propagators is compatible (or can be entangled) if the associated binary partitions can consistently be oriented, i.e., if all the internal lines contribute to the entangled cuts with the same orientation.

4. Causal propagator orientation: When external particles are present, the relative signs among aligned on-shell energies (i.e., $\sum q_{i, 0}^{(+)}$) and the energy component of the external momenta is determined by the orientation matrix, which can be built from the vertex matrix.

In Figure 18, we show examples of causal propagators which cannot be entangled for the Mercedes-Benz diagram (i.e., a particular case of $\mathrm{N}^{2} \mathrm{MLT}$ or a four-vertex topology). On the left, the criteria 2 is not fulfilled, since the associated partitions of vertices are not disjoint. 
On the right, we consider three causal propagators which cannot be consistently oriented (criteria 3): the edge connecting the vertices 1 and 3 does not allow a compatible orientation.
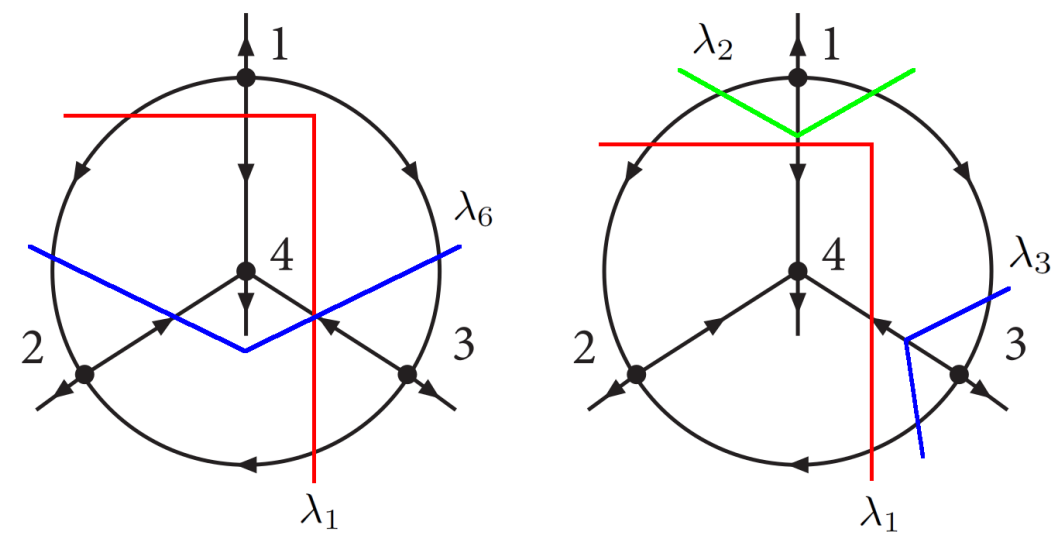

Figure 18. Example of two incompatible causal propagators with nondisjoint sets of vertices (left) and incompatible momenta orientation (right).

Thus, given a diagram, determining its vertex matrix and imposing the selection criteria $1-4$, we can obtain a formula describing its causal structure. In accordance with the results presented in Ref. [100], we found that

$$
\mathcal{A}_{N}^{(L)}(1, \ldots, L+k)=\sum_{\sigma \in \Sigma} \int_{\vec{\ell}_{1}, \cdots, \vec{\ell}_{L}} \frac{\mathcal{N}_{\sigma}\left(\left\{q_{r, 0}^{(+)}\right\},\left\{p_{j, 0}\right\}\right)}{x_{L+k}} \times \prod_{i=1}^{k} \frac{1}{-\lambda_{\sigma(i)}}+(\sigma \leftrightarrow \bar{\sigma}),
$$

describes the causal structure of any multi-loop multi-leg Feynman diagram or collection of Feynman diagrams with topological complexity $k-1$. The set $\Sigma$ contains all the subsets of products of $k$ causal propagators $\lambda_{i}^{ \pm}$fulfilling the selection criteria $1-4$, and $\mathcal{N}_{\sigma}$ is given by the application of an operator depending on the subset of $\sigma$. Thus, this result confirms that multi-loop multi-leg amplitudes can be purely described in terms of vertices and edges, regardless the number of loops or external lines.

\section{Four-Dimensional Unsubtraction (FDU)}

The standard computation of accurate predictions at Next-to-Leading Order (NLO) in QFT needs to deal with non-trivial integrands. These integrands carry multiple singularities due to the low (IR) and high (UV) energy regimes. The cancellation of those divergent quantities typically occurs at integral level. By considering the Kinoshita-Lee-Nauenberg (KLN) theorem [113,114], soft and collinear divergences (IR singularities) are removed, and by adding suitable ultraviolet counter-terms, UV singularities are renormalized, rendering the cross-section finite. This algorithm for cancelling singularities can be extended to Next-to-Next-to-Leading order (NNLO) and beyond; however, since the complexity of the integrands is higher at higher orders, this procedure is reaching a bottleneck because cancellation of singularities must be achieved at integral level, i.e., the $\epsilon \rightarrow 0$ limit is taken once the cross-section is known in DREG. Since LTD transform loop integrals in phase-space integrals, among other properties discussed in this document, the cancellation of singularities could occur at integrand level. Hence, based on the LTD theorem, the Four-Dimensional Unsubtraction [82-85,111] (FDU) method presents a new paradigm to compute observables in four dimensions, since the definition of cross-section is free of IR and UV singularities. The first application of the LTD at cross-section level was done in a toy model based on the simplest $\phi^{3}$ theory. In Ref. [82] it was shown for the first time that LTD is powerful to build physical observables in four dimensions by a proper mapping between real and virtual kinematical variables. 


\subsection{Local Cancellation of Infrared Singularities within FDU}

Let us start the discussion of the FDU approach by analyzing the cancellation of soft and collinear divergences. The simplest scenario is the decay process $1 \rightarrow n$ where the Born level cross-section is given by,

$$
\sigma^{(0)}=\int \operatorname{dPS}^{1 \rightarrow n}\left|\mathcal{M}_{n}^{(0)}\right|^{2} \mathcal{S}_{0}\left(\left\{p_{i}\right\}\right),
$$

with $\left|\mathcal{M}_{n}^{(0)}\right|^{2}$ the LO contribution and $\mathcal{S}_{0}$ is the IR-safe measure function. The virtual correction to the LO cross-section is computed as

$$
\sigma_{V}^{(1)}=\int \operatorname{dPS}^{1 \rightarrow n} \int_{\ell} 2 \operatorname{Re}\left\langle\mathcal{M}_{n}^{(0)} \mid \mathcal{M}_{n}^{(1)}\right\rangle \mathcal{S}_{0}\left(\left\{p_{i}\right\}\right),
$$

where $\left\langle\mathcal{M}_{n}^{(0)} \mid \mathcal{M}_{n}^{(1)}\right\rangle$ represents the interference between the Born level and one-loop amplitudes. It is important to emphasize at this point that FDU requires also to maintain self-energy contributions because they contain both IR and UV divergences that will contribute to the full cancellation of singularities. The IR singularities from the virtual component are cancelled against real-radiation contributions.

For the sake of simplicity, let us consider that the process under study only has final-state radiation singularities. Hence, the real contribution given by

$$
\sigma_{R}^{(1)}=\int \operatorname{dPS}^{1 \rightarrow n+1}\left|\mathcal{M}_{n+1}^{(0)}\right|^{2} \mathcal{S}_{1}\left(\left\{p_{i}^{\prime}\right\}\right),
$$

contains all Feynman diagrams with one extra particle in the final state in $\mathcal{M}_{n+1}^{(0)}$ and $\mathcal{S}_{1}$ is the measure function for $n+1$ particles. It is important to recall at this moment that primed variables are used to describe the momenta of real matrix elements while unprimed variables are labeling Born and loop level momenta.

The FDU starts with the analysis of the virtual cross-section which can be decomposed as a sum of dual contributions as,

$$
\begin{aligned}
\sigma_{V}^{(1)} & =\int \mathrm{dPS}^{1 \rightarrow n} \sum_{i=1}^{N} \int_{\vec{\ell}} I_{i}\left(q_{i}\right) \mathcal{S}_{0}\left(\left\{p_{i}\right\}\right) \\
& \equiv \int \mathrm{dPS}^{1 \rightarrow n} \sum_{i=1}^{N} \sigma_{D, i}^{(1)},
\end{aligned}
$$

with $I_{i}\left(q_{i}\right)$ the $N$ dual integrals that arise from the direct application of the LTD theorem. We notice that each $I_{i}\left(q_{i}\right)$ has set one internal particle on-shell and it is characterized by $q_{i}$. Then, the loop integral has been transformed to phase-space integrals, therefore each dual integral behaves as the contribution of one real particle emitted. To achieve the complete cancellation of IR singularities, each $\sigma_{D, i}^{(1)}$ must be paired, at integrand level with a corresponding real-radiation twin component. The real radiation is sliced as,

$$
\sigma_{R, i}^{(1)}=\int \mathrm{dPS}^{1 \rightarrow n+1} d \sigma_{R}^{(1)} \mathcal{R}_{i},
$$

where $\mathcal{R}_{i}$ represents a partition of the full integral, such that $\sum_{i} \sigma_{R, i}^{(1)}=\sigma_{R}^{(1)}$ and considering that only one IR divergent configuration is allowed in each $\mathcal{R}_{i}^{\prime}$. Finally, to merge both integrals, a mapping between the kinematical variables is implemented. On the one hand, each partition in $\sigma_{D, i}^{(1)}$ contains $n$ external momenta plus one extra integration variable corresponding to $q_{i}$, therefore, each $\sigma_{D, i}^{(1)}$ is mapped into its real sliced contribution as,

$$
\mathcal{T}_{i}\left(\left\{p_{1}, \ldots, p_{n}, q_{i}\right\}\right) \rightarrow\left\{p_{1}^{\prime}, \ldots, p_{n+1}^{\prime}\right\},
$$

where $\mathcal{T}_{i}$ is a bijective transformation among real and virtual variables. The momentum mapping between variables is analogous to the one used by the dipole method $[57,58]$, since 
the singularities are associated with soft emissions or the double collinear limit $[115,116]$. These singular IR configurations are associated with specific contributions in the virtual part, by selecting two massless partons per partition. On one hand the spectator and on the other hand the emitter. Hence, the four momenta of the emitter and spectator in addition with the loop-three momentum are used to reconstruct the kinematical phase-space of the real-emission cross-section where there is a similar configuration, i.e., an emitter decaying into two partons in a soft or collinear regime. Explicitly, if $p_{i}$ is the momentum of the finalstate emitter, $q_{i}$ the internal on-shell momentum prior the emitter and $p_{j}$ is the momentum of the final-state spectator, we apply the momentum mapping

$$
\begin{aligned}
& p_{r}^{\prime \mu}=q_{i}^{\mu}, \\
& p_{i}^{\prime \mu}=p_{i}^{\mu}-q_{i}^{\mu}+\alpha_{i} p_{j}^{\mu}, \\
& p_{j}^{\prime \mu}=\left(1-\alpha_{i}\right) p_{j}^{\mu}, \quad k \neq i, j, \\
& p_{k}^{\prime \mu}=p_{k}^{\mu}, \quad
\end{aligned}
$$

with $p_{r}^{\prime}$ the momentum of the extra radiation of the process and, in the massless scenario, $\alpha_{i}=\left(q_{i}-p_{i}\right)^{2} /\left(2 p_{j} \cdot\left(q_{i}-p_{i}\right)\right)$. Furthermore, this mapping preserves momentum conservation since $p_{i}+p_{j}+\sum_{k \neq i, j} p_{k}=p_{i}^{\prime}+p_{j}^{\prime}+p_{r}^{\prime}+\sum_{k \neq i, j} p_{k}^{\prime}$ is fulfilled. A similar mapping is used when considering massive particles and it was shown in Ref [85]. The extension of this formalism to higher orders (i.e., NNLO and beyond) will require additional kinematical transformations which take care of the singular behavior of scattering amplitudes in the multiple-collinear limit [117-123].

\subsection{Self-Energy Insertions and Renormalization}

Before moving forward with IR cancellation, let us review the local renormalization of UV singularities. The standard cancellation of UV singularities requires the renormalization of field wave-functions and couplings. In the FDU formalism, this feature is obtained through the construction of local UV counter-terms. At one loop, the scenario where massless and massive particles are propagating in the loop has been studied [84,85]. Remarkably, it was shown that a smooth transition between massless and massive renormalization constants takes place and UV singularities are well understood. Let us highlight a crucial difference between the standard renormalization constant in DREG and LTD. Wave-function renormalization constants are obtained from self-energy diagrams. In particular, massless bubble diagrams in DREG are neglected in the renormalization procedure since IR and UV divergences are considered to be equal and they cancel out. However, the same divergent poles in the FDU formalism contribute separately. Specifically, it means that IR singularities of loop diagrams vanish with only the IR poles of the real radiation and there are no mixed cancellation between UV and IR poles. Therefore, the remaining UV divergences must be removed when renormalization is implemented in the FDU scheme. Hence, we stress that integrands in the FDU are separated into the IR and UV domains, and this identification is crucial to render cross-sections free of singularities in the FDU formalism.

We analyze the construction of the renormalization constants in the FDU framework. Using standard Feynman rules, the unintegrated massive wave-function renormalization constant, in the Feynman gauge, at one loop is given by,

$$
\Delta Z_{2}\left(p_{1} ; M\right)=-g_{\mathrm{S}}^{2} C_{\mathrm{F}} \int_{\ell} G_{F}\left(q_{1}\right) G_{F}\left(q_{3}\right)\left[(d-2) \frac{q_{1} \cdot p_{2}}{p_{1} \cdot p_{2}}+4 M^{2}\left(1-\frac{q_{1} \cdot p_{2}}{p_{1} \cdot p_{2}}\right) G_{F}\left(q_{3}\right)\right] .
$$

Equation (64) is the most general wave-function renormalization constant since it includes the massless and massive case and, as previously mentioned, the transition to the massless case, $\Delta Z_{2}(p, 0)$, is straightforward. The function $\Delta Z_{2}\left(p_{1} ; M\right)$ contains singularities associated with the UV domain, therefore we must find the UV component of Equation (64), $\Delta \mathrm{Z}_{2}^{\mathrm{UV}}$, and subtract it in order to find a UV-free wave-function renormalization constant, 
$\Delta Z_{2}^{\mathrm{IR}}$. The UV part is extracted by performing an expansion of the integrand around the $\mathrm{UV}$ propagator $G_{F}\left(q_{\mathrm{UV}}\right)=\left(q_{\mathrm{UV}}^{2}-\mu_{\mathrm{UV}}^{2}+\imath 0\right)^{-1}$. In particular, for Equation (64), it is found

$$
\Delta \mathrm{Z}_{2}^{\mathrm{UV}}\left(p_{1}\right)=(2-d) g_{\mathrm{S}}^{2} C_{\mathrm{F}} \int_{\ell}\left[G_{F}\left(q_{\mathrm{UV}}\right)\right]^{2}\left(1+\frac{q_{\mathrm{UV}} \cdot p_{2}}{p_{1} \cdot p_{2}}\right)\left[1-G_{F}\left(q_{\mathrm{UV}}\right)\left(2 q_{\mathrm{UV}} \cdot p_{1}+\mu_{\mathrm{UV}}^{2}\right)\right] .
$$

Hence, we define $\Delta Z_{2}^{\mathrm{IR}}$ as

$$
\Delta Z_{2}^{\mathrm{IR}}=\Delta \mathrm{Z}_{2}-\Delta \mathrm{Z}_{2}^{\mathrm{UV}}
$$

which is free of UV singularities and the IR singularities poles are those needed to remove the remaining singularities from the virtual and real mapping of the cross-section.

To remove UV singularities, it is important to build proper UV counter-terms that can be extracted by the direct study of the UV properties of physical amplitudes. After the combination of real and virtual matrix elements and renormalization, the remaining amplitude has only UV singularities, in the following, we consider generic amplitudes with only UV singularities. At two loops, a generic two-loop amplitude can be written as,

$$
\mathcal{A}^{(2)}=\int_{\ell_{1}} \int_{\ell_{2}} \mathcal{I}\left(\ell_{1}, \ell_{2}\right),
$$

where the integrand is a function of the loop variables $\ell_{1}$ and $\ell_{2}$. UV divergences shall appear when the three-momenta $\left|\vec{\ell}_{1}\right|$ and $\left|\vec{\ell}_{2}\right|$ tend to infinity. In the two-loop scenario, there are three possible UV limits: (i) $\left|\vec{\ell}_{1}\right| \rightarrow \infty$ and $\left|\vec{\ell}_{2}\right|$ remains fixed, (ii) $\left|\vec{\ell}_{2}\right| \rightarrow \infty$ and $\left|\vec{\ell}_{1}\right|$ is fixed and, (iii) $\left|\vec{\ell}_{1,2}\right| \rightarrow \infty$. To extract the UV behavior of the integrand, we impose the replacement,

$$
\mathcal{S}_{j, \mathrm{UV}}:\left\{\ell_{j}^{2} \mid \ell_{j} \cdot k_{i}\right\} \rightarrow\left\{\lambda^{2} q_{j, \mathrm{UV}}^{2}+\left(1-\lambda^{2}\right) \mu_{\mathrm{UV}}^{2} \mid \lambda q_{j, \mathrm{UV}} \cdot k_{i}\right\},
$$

for a given loop momentum $\ell_{j}$ and then, we expand the expression up to logarithmic order around the UV propagator. This action is represented by the $L_{\lambda}$ operator. Notice, however, that the result shall generate a finite part after integration that has to be fixed to find the right value of the integral. Therefore, the first counter-terms is computed by

$$
\mathcal{A}_{j, \mathrm{UV}}^{(2)}=L_{\lambda}\left(\left.\mathcal{A}^{(2)}\right|_{\mathcal{S}_{j, \mathrm{UV}}}\right)-d_{j, \mathrm{UV}} \mu_{\mathrm{UV}}^{2} \int_{\ell_{j}}\left(G_{F}\left(q_{j, \mathrm{UV}}\right)\right)^{3},
$$

with $d_{j, \mathrm{UV}}$ the fixing parameter which makes the finite part of integral to be zero in the $\overline{\mathrm{MS}}$ scheme.

The remaining divergences shall occur when both $\left|\vec{\ell}_{1}\right|$ and $\left|\vec{\ell}_{2}\right|$ approach to infinity simultaneously. Then, to build a counter-term that mimics this behavior, the following replacement is implemented,

$$
\begin{aligned}
\mathcal{S}_{\mathrm{UV}^{2}}: & \left\{\ell_{j}^{2}\left|\ell_{j} \cdot \ell_{k}\right| \ell_{j} \cdot k_{i}\right\} \rightarrow \\
& \left\{\lambda^{2} q_{j, \mathrm{UV}}^{2}+\left(1-\lambda^{2}\right) \mu_{\mathrm{UV}}^{2}\left|\lambda^{2} q_{j, \mathrm{UV}} \cdot q_{k, \mathrm{UV}}+\left(1-\lambda^{2}\right) \mu_{\mathrm{UV}}^{2} / 2\right| \lambda q_{j, \mathrm{UV}} \cdot k_{i}\right\}
\end{aligned}
$$

on the subtracted integrand. As with the previous UV counter-term, the application of the $L_{\lambda}$ operation shall produce a finite piece that has to be fixed to build properly the counter-term, $\mathcal{A}_{\mathrm{UV}^{2}}^{(2)}$. Explicitly,

$$
\mathcal{A}_{\mathrm{UV}^{2}}^{(2)}=L_{\lambda}\left(\left.\left(\mathcal{A}^{(2)}-\sum_{j=1,2} \mathcal{A}_{j, \mathrm{UV}}^{(2)}\right)\right|_{\mathcal{S}_{\mathrm{UV}^{2}}}\right)-d_{\mathrm{UV}^{2}} \mu_{\mathrm{UV}}^{4} \int_{\ell_{1}} \int_{\ell_{2}}\left(G_{F}\left(q_{1, \mathrm{UV}}\right)\right)^{3}\left(G_{F}\left(q_{12, \mathrm{UV}}\right)\right)^{3},
$$


with $d_{\mathrm{UV}^{2}}$ the fixing parameter of the double UV limit. With all UV divergences under control, the renormalized amplitude at two loops, $\mathcal{A}_{\mathrm{R}}^{(2)}$, can be constructed by the subtraction of all UV counter-terms, such that

$$
\mathcal{A}_{\mathrm{R}}^{(2)}=\mathcal{A}^{(2)}-\mathcal{A}_{1, \mathrm{UV}}^{(2)}-\mathcal{A}_{2, \mathrm{UV}}^{(2)}-\mathcal{A}_{\mathrm{UV}}^{(2)},
$$

is free of IR and UV singularities.

In the multi-loop scenario, multiple ultraviolet poles will appear, since all loops could tend to infinity at different speed.

\section{3. $A^{*} \rightarrow q \bar{q}$ at $\mathrm{NLO}$ in $\mathrm{QCD}$}

The FDU method has been studied in different processes, for the sake of simplicity, we recall the application of the algorithm in $A^{*} \rightarrow q \bar{q}$ a NLO in QCD, with $A=H, \gamma, Z$.

At NLO, virtual correction $A^{*}(p) \rightarrow q\left(p_{1}\right)+\bar{q}\left(p_{2}\right)$ must be mapped into the realradiation process $A^{*}(p) \rightarrow q\left(p_{1}^{\prime}\right)+\bar{q}\left(p_{2}^{\prime}\right)+g\left(p_{r}^{\prime}\right)$. As explained in previous subsections, we must also keep all virtual contributions since self-energy contributions are crucial to cancel out all IR singularities. In Figure 19, we present the complete set of Feynman diagrams of the real and virtual processes.
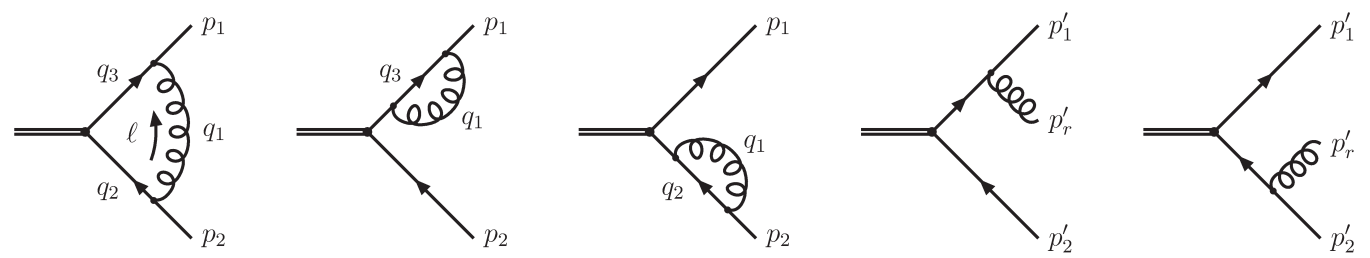

Figure 19. Momentum configuration of NLO QCD corrections to the process $A^{*} \rightarrow q \bar{q}(q)$, assuming that the decaying particle does not couple to gluons.

It is important to recall that in this example we consider massive quarks, i.e., $p_{1}^{2}=p_{2}^{2}=M^{2}$ and $p_{1}^{\prime 2}=p_{2}^{\prime 2}=M^{2}$ and $p_{r}^{\prime 2}=0$. It is convenient to write the momenta $p_{1}$ and $p_{2}$ in terms of massless four vectors, $\hat{p}_{1}$ and $\hat{p}_{2}$, such as

$$
p_{1}^{\mu}=\beta_{+} \hat{p}_{1}^{\mu}+\beta_{-} \hat{p}_{2}^{\mu}, \quad p_{2}^{\mu}=\beta_{-} \hat{p}_{1}^{\mu}+\beta_{+} \hat{p}_{2}^{\mu},
$$

with $\beta_{ \pm}=(1 \pm \beta) / 2, \beta=\sqrt{1-m^{2}}$ and $m=2 M / \sqrt{s_{12}}$. Therefore, since the momentum of the gluon is labeled in the virtual contribution as $q_{1}$ and LTD shall set it on-shell, $q_{1}^{2}=0$, the momentum mapping proposed for the merging of virtual and real cross-sections is

$$
\begin{aligned}
& p_{r}^{\prime \mu}=q_{1}^{\mu}, \\
& p_{1}^{\prime \mu}=\left(1-\alpha_{1}\right) \hat{p}_{1}^{\mu}+\left(1-\gamma_{1}\right) \hat{p}_{2}^{\mu}-q_{1}^{\mu}, \\
& p_{2}^{\prime \mu}=\alpha_{1} \hat{p}_{1}^{\mu}+\gamma_{1} \hat{p}_{2}^{\mu},
\end{aligned}
$$

where $\alpha_{1}$ and $\gamma_{1}$ are parameters determined by imposing on-shellness. Notice that Equation (74) fulfills momentum conservation. This mapping will be used to remove some IR singularities of the integral related to $\tilde{\delta}\left(q_{1}\right)$, the remaining IR singularities shall cancel through renormalization. Now, since there are other singular IR behaviors in the real that can be recognized in the virtual, when $q_{2}^{2}=M^{2}$, the second momentum mapping is proposed to be,

$$
\begin{aligned}
& p_{r}^{\prime \mu}=\left(1-\gamma_{2}\right) \hat{p}_{1}^{\mu}+\left(1-\alpha_{2}\right) \hat{p}_{2}^{\mu}-q_{2}^{\mu}, \\
& p_{1}^{\prime \mu}=\gamma_{2} \hat{p}_{1}^{\mu}+\alpha_{2} \hat{p}_{2}^{\mu}, \\
& p_{2}^{\prime \mu}=q_{2}^{\mu},
\end{aligned}
$$


with $\alpha_{2}$ and $\gamma_{2}$ parameters which are also determined by the on-shell conditions. In virtue of soft and collinear divergences in the real cross-section being only in these two LTD cuts, we proceed to slice the full real element with the general function,

$$
\mathcal{R}_{i}=\left\{y_{i r}^{\prime}<\min \left(y_{j k}^{\prime}\right)\right\}, \quad \sum \mathcal{R}_{i}=1,
$$

which in this particular case are explicitly

$$
\theta\left(y_{2 r}^{\prime}<y_{1 r}^{\prime}\right)+\theta\left(y_{1 r}^{\prime}<y_{2 r}^{\prime}\right)=1,
$$

with $y_{i r}^{\prime}=2 p_{i}^{\prime} \cdot p_{r}^{\prime} / s_{12}$. The integration domain is well determined, and it is shown in Figure 20.

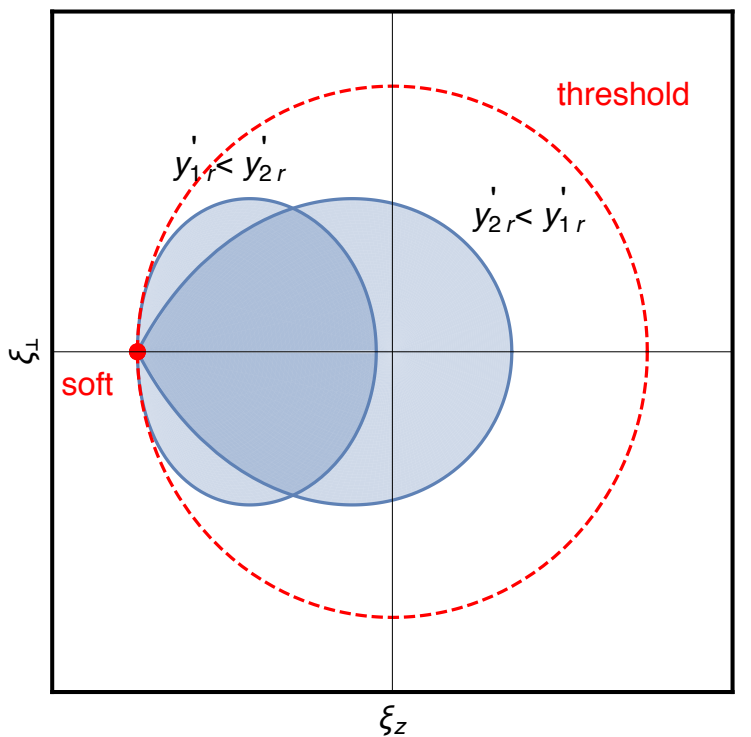

Figure 20. The dual integration regions in the loop-three momentum space, with $\xi_{\perp}=\sqrt{\xi_{x}^{2}+\xi_{y}^{2}}$.

We remark that this arrangement of the real element phase-space is general, and it can be applied to any $1 \rightarrow 3$ real cross-section that is mapped into the $1 \rightarrow 2$ virtual cross-section.

Let us now compute the virtual decay rate of $A^{*} \rightarrow q \bar{q}$. The LTD representation is given by,

$$
\Gamma_{\mathrm{V}, A}^{(1, \mathrm{R})}=\frac{1}{2 \sqrt{s_{12}}} \sum_{i=1}^{3} \int \mathrm{dPS}^{1 \rightarrow 2} 2 \operatorname{Re}\left\langle\mathcal{M}_{A}^{(0)} \mid \mathcal{M}_{A}^{(1, \mathrm{R})}\left(\widetilde{\delta}\left(q_{i}\right)\right)\right\rangle
$$

with the renormalized one-loop amplitude computed as,

$$
\left|\mathcal{M}_{A}^{(1, \mathrm{R})}\right\rangle=\left|\mathcal{M}_{A}^{(1)}\right\rangle-\left|\mathcal{M}_{A}^{(1, \mathrm{UV})}\right\rangle+\frac{1}{2}\left(\Delta \mathrm{Z}_{2}^{\mathrm{IR}}\left(p_{1}\right)+\Delta Z_{2}^{\mathrm{IR}}\left(p_{2}\right)\right)\left|\mathcal{M}_{A}^{(0)}\right\rangle,
$$

and $\left|\mathcal{M}_{A}^{(1, \mathrm{UV})}\right\rangle$ is the unintegrated UV counter-term of the one-loop vertex correction. The real element, after the splitting of the phase-space in two domains, is written as,

$$
\widetilde{\Gamma}_{\mathrm{R}, A, i}^{(1)}=\frac{1}{2 \sqrt{s_{12}}} \int \mathrm{dPS}^{1 \rightarrow 3}\left|\mathcal{M}_{A^{*} \rightarrow q \bar{g} g}^{(0)}\right|^{2} \mathcal{R}_{i}\left(y_{i r}^{\prime}<y_{j r}^{\prime}\right), \quad i, j=\{1,2\} .
$$

The sum of the virtual and real contributions in Equations (78) and (80) is a single integral in the loop-three momentum. It is UV and IR finite locally and can be calculated numerically with $\epsilon=0$. The results, normalized to the LO decay rate $\Gamma_{A}^{(0)}$, are presented in Figures 21 and 22. The agreement with the analytical predictions is excellent in all cases. 
Furthermore, we notice that the massless quark scenario is recovered smoothly in the FDU however, in DREG this is not the case since individual contributions are not smoothly defined in that limit.

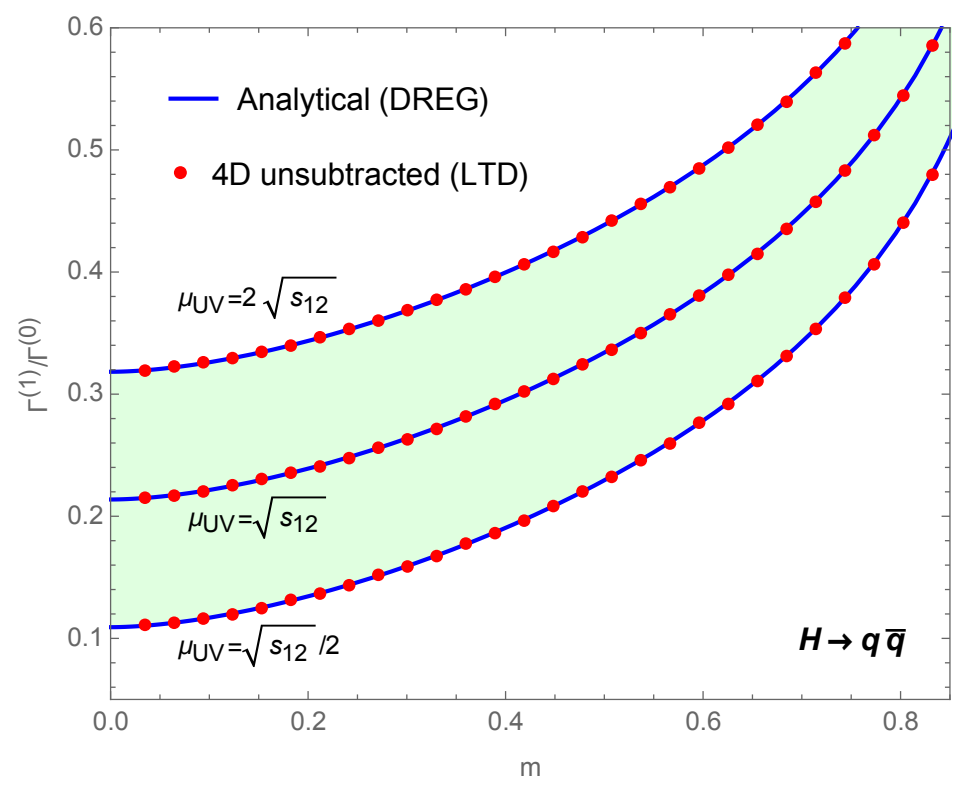

Figure 21. NLO total decay rate for $H^{*} \rightarrow q \bar{q}$ normalized to its corresponding LO decay rate. The solid lines correspond to DREG analytic result while dots were computed numerically in the FDU formalism for the standard Higgs boson.

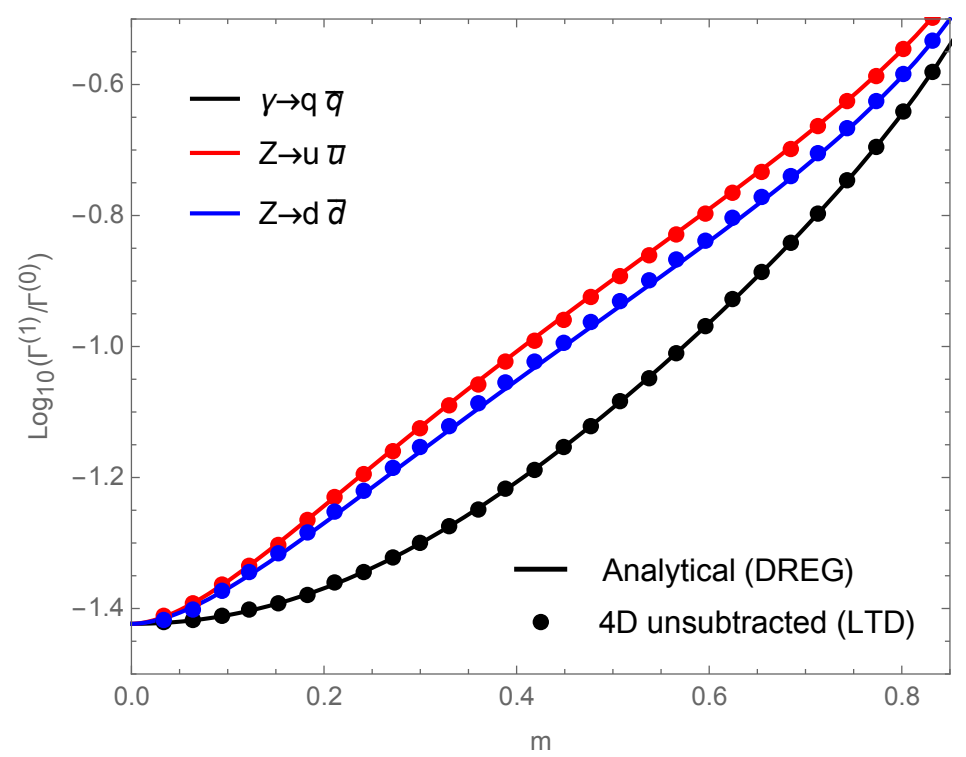

Figure 22. NLO total decay rate for $V^{*} \rightarrow q \bar{q}$ normalized to its corresponding LO decay rate. The solid lines correspond to DREG analytic result while dots were computed numerically in the FDU formalism for off-shell vector bosons.

\section{Local Dual Counter-Terms for Cross-Sections}

In the following, we will focus on the NLO QCD corrections to cross-sections involving massless colored particles and any number of other colorless SM particles. The extension of the following ideas to the massive case has been worked out following the same reasoning, which is presented in Ref. [86]. The reduction of any one-loop amplitude to a combination of scalar integrals is an easy task compared to the two-loop case. Of course, such a reduction does not alter the structure of the divergences of the amplitude but to properly reproduce 
all the IR singularities, in addition to the wave-function renormalization, one must keep also the massless bubble integrals that are formally discarded usually (as already explained in Section 8). Once all the box functions are expressed in terms of their corresponding 6-dimensional version, it turns out that the IR divergences of a renormalized one-loop amplitude interfered with the leading order are given by the triangle scalar functions with two external partons connected by a massless propagator times the corresponding color connected tree level amplitude multiplied $s_{i j}=2 p_{i} \cdot p_{j}$, the massless bubbles times $(1 / 2)$ the Born for every external (gluon) quark external particle multiplied times the corresponding Casimir, and the external wave-function renormalization constant time the Born. The above set of IR singularities is universal and, in fact, it reproduces the known formula for the poles of any one-loop amplitude in QCD. Inspired by the LTD theorem, we considered the cuts of these loop integrals that capture their infrared and collinear divergences. As a result of this procedure one obtains a novel subtraction scheme. For the sake of simplicity we will restrict the discussion to the case of a colorless initial state and so we will consider colored radiation exchanged only among the final-state partons. Our general formula for the dual counter-term in the case of a quark emitting a gluon is the following:

$\sigma_{q g, b}^{D S} \equiv 8 \pi \alpha_{S} \mathcal{N}_{i n} \int d \Phi_{m}\left\langle 1, \ldots, m\left|\mathbf{T}_{a c} \mathbf{T}_{b}\right| 1, \ldots, m\right\rangle_{m}\left[V_{q_{a g_{c}, b}}\left(p_{i}, p_{j}\right)+G_{q_{a g_{c}, b}}\left(p_{i}, p_{j}\right)\right]$,

where $\mathcal{N}_{i n}$ represents all the non-QCD factors, including the symmetry factor for identical partons in the final state, $|1, \ldots, m\rangle_{m}$ is the $m$-parton Born amplitude and $\mathbf{T}_{a c} \mathbf{T}_{b}$ is a colorcharge operator acting on the color indices of the partons $a c$ and $b$, respectively. The functions $V_{q g, b}$ and $G_{q g, b}$ in Equation (81) are defined by

$$
\begin{aligned}
V_{q g, b} & \equiv \int_{q_{1}} \tilde{\delta}\left(q_{1}\right) \mathcal{R}_{1}\left[-\frac{2 s_{i j}}{\left(-2 q_{1} \cdot p_{i}\right)\left(2 q_{1} \cdot p_{j}\right)}+\frac{2}{\left(-2 q_{1} \cdot p_{i}\right)}\right] \\
G_{q g, b} & \equiv-\frac{(1-\varepsilon)}{s_{i j}} \int_{q_{1}} \tilde{\delta}\left(q_{1}\right) \mathcal{R}_{1} \frac{2 q_{1} \cdot p_{j}}{\left(-2 q_{1} \cdot p_{i}\right)}
\end{aligned}
$$

where $V_{q g, b}$ groups together the dual contributions from the reduction of the virtual amplitude and $G_{q g, b}$ is the contribution of the quark wave-function renormalization. In the above formulae $p_{i}$ and $p_{j}$ represent the momentum of the emitter (with color index $a c$ ) and spectator (color index $b$ ) in the Born kinematic respectively, while $q_{1}$ is the loop momentum that is associated with the gluon in the real-radiation kinematic. In the case a gluon is radiated collinear to another gluon the corresponding dual counter-term $\sigma_{g g, b}^{D S}$ is given by

$$
\sigma_{g g, b}^{D S} \equiv 8 \pi \alpha_{S} \mathcal{N}_{i n} \int d \Phi_{m m}\left\langle 1, \ldots, m\left|\mathbf{T}_{a c} \mathbf{T}_{b}\left[V_{g g, b}^{\mu v}\left(p_{i}, p_{j}\right)+G_{g g, b}^{\mu v}\left(p_{i}, p_{j}\right)\right]\right| 1, \ldots, m\right\rangle_{m},
$$

where the symmetrization $p_{a}^{\prime} \leftrightarrow p_{c}^{\prime}$ must be performed on the right-hand side. Once again, in Equation (83) we have extracted only the dual contributions with $q_{1}$ on-shell, obtaining

$$
\begin{aligned}
V_{g g, b}^{\mu v} & \equiv-\int_{q_{1}} \tilde{\delta}\left(q_{1}\right) \mathcal{R}_{1}\left[-\frac{2 s_{i j}}{\left(-2 q_{1} \cdot p_{i}\right)\left(2 q_{1} \cdot p_{j}\right)}+\frac{1}{\left(-2 q_{1} \cdot p_{i}\right)}\right] g^{\mu v}, \\
G_{g g, b}^{\mu v} & \equiv-\int_{q_{1}} \frac{\tilde{\delta}\left(q_{1}\right) \mathcal{R}_{1}}{\left(-2 q_{1} \cdot p_{i}\right)}\left[g^{\mu v}+\frac{d-2}{\left(-2 q_{1} \cdot p_{i}\right)}\left(\frac{q_{1} \cdot p_{j}}{p_{i} \cdot p_{j}}-1\right)\left(q_{1}^{\mu}-\frac{q_{1} \cdot p_{i}}{p_{i} \cdot p_{j}} p_{j}^{\mu}\right)\left(q_{1}^{v}-\frac{q_{1} \cdot p_{i}}{p_{i} \cdot p_{j}} p_{j}^{v}\right)\right] .
\end{aligned}
$$

Finally, the case of a gluon splitting into a collinear quark-antiquark pair receive contributions only from the wave-function contribution so that the corresponding dual counter-term $\sigma_{q \bar{q}, b}^{D S}$ is given by

$$
\sigma_{q \bar{q}, b}^{D S} \equiv 8 \pi \alpha_{S} \mathcal{N}_{i n} \int d \Phi_{m m}\left\langle 1, \ldots, m\left|\mathbf{T}_{a c} \mathbf{T}_{b} G_{q \bar{q}, b}^{\mu v}\left(p_{i}, p_{j}\right)\right| 1, \ldots, m\right\rangle_{m}
$$

with 


$$
G_{q \bar{q}, b}^{\mu \nu} \equiv \int_{q_{1}} \frac{\tilde{\delta}\left(q_{1}\right) \mathcal{R}_{1} T_{R} N_{f}}{C_{A}\left(-2 q_{1} \cdot p_{i}\right)}\left[g^{\mu \nu}+\frac{4}{\left(-2 q_{1} \cdot p_{i}\right)}\left(\frac{q_{1} \cdot p_{j}}{p_{i} \cdot p_{j}}-1\right)\left(q_{1}^{\mu}-\frac{q_{1} \cdot p_{i}}{p_{i} \cdot p_{j}} p_{j}^{\mu}\right)\left(q_{1}^{v}-\frac{q_{1} \cdot p_{i}}{p_{i} \cdot p_{j}} p_{j}^{v}\right)\right] .
$$

Once a proper momentum mapping is considered, these counter-terms can be analytically integrated over the region in which $p_{a} \cdot p_{c}<p_{b} \cdot p_{c}$ in the real phase-space and added back to the virtual contribution. This last operation corresponds to extract all the infrared and collinear divergences out of the virtual matrix element. We have used the mapping adopted in the seminal work by Catani and Seymour [58].

$\gamma^{*} \rightarrow 3$ Jets at $\mathrm{NLO}$

As an example of application, we consider the NLO correction to the three-jet production in $e^{+} e^{-}$annihilation $\gamma^{*} \rightarrow q\left(p_{1}\right) \bar{q}\left(p_{2}\right) g\left(p_{3}\right)$. For simplicity we will restrict to the case of gluon radiation, namely $\gamma^{*} \rightarrow q\left(p_{1}^{\prime}\right) \bar{q}\left(p_{2}^{\prime}\right) g\left(p_{3}^{\prime}\right) g\left(p_{4}^{\prime}\right)$. The case of four quark production can be treated along the same lines. By inspecting the virtual sector, we find six emitter-spectator pairs for the process $\gamma^{*} \rightarrow q\left(p_{1}\right) \bar{q}\left(p_{2}\right) g\left(p_{3}\right)$ that are $\{q \bar{q}, q g, \bar{q} g\}$ and the three pairs where the emitter and the spectator switch. Once a real kinematic configuration $\left(p_{1}^{\prime}, p_{2}^{\prime}, p_{3}^{\prime}, p_{4}^{\prime}\right)$ is generated, the six kinematic invariants $s_{i j}$ are analyzed and as a consequence six dual counter-terms are activated. To each emitter-spectator pair, we associate the following dual counter-terms:

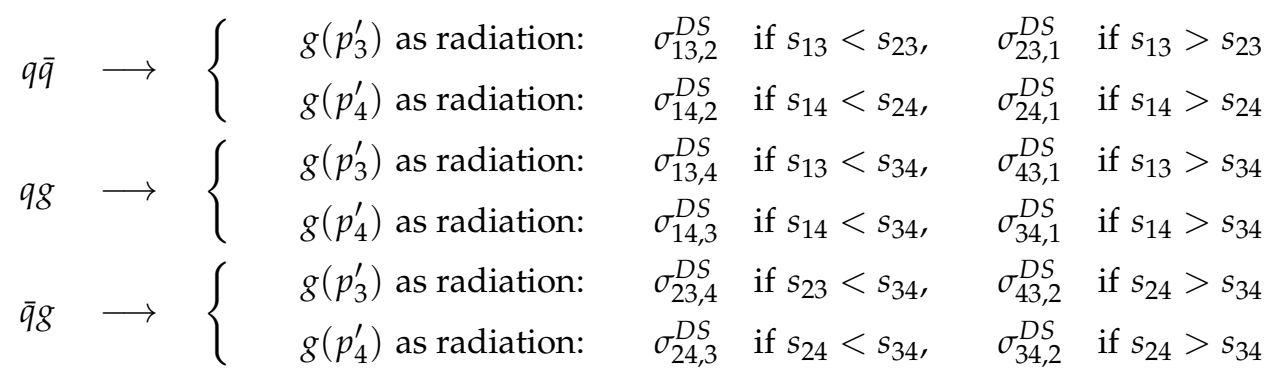

Since the process involves only three colored partons, the color algebra factorizes and one has $\mathbf{T}_{1} \mathbf{T}_{2}=C_{A} / 2-C_{F}$ and $\mathbf{T}_{1} \mathbf{T}_{3}=\mathbf{T}_{2} \mathbf{T}_{3}=-C_{A} / 2$. In the virtual sector, we use the integrated version of the dual counter-terms that are given by

$$
\begin{aligned}
\sigma_{q, \bar{q}}^{D S} & \sim \frac{\alpha_{S}}{2 \pi} \frac{(4 \pi)^{\varepsilon}}{\Gamma(1-\varepsilon)}\left(\frac{C_{A}}{2}-C_{F}\right) \int d \Phi_{2}\left(\frac{\mu^{2}}{s_{12}}\right)^{\varepsilon}\left|A_{q \bar{q} g}^{(0)}\right|^{2}\left[\frac{1}{\varepsilon^{2}}+\frac{3}{2 \varepsilon}+3+4 \log (2)-\frac{\pi^{2}}{2}\right] \\
\sigma_{\bar{q}, \bar{q}}^{D S} & \sim \frac{\alpha_{S}}{2 \pi} \frac{(4 \pi)^{\varepsilon}}{\Gamma(1-\varepsilon)}\left(\frac{C_{A}}{2}-C_{F}\right) \int d \Phi_{2}\left(\frac{\mu^{2}}{s_{12}}\right)^{\varepsilon}\left|A_{q \bar{q} g}^{(0)}\right|^{2}\left[\frac{1}{\varepsilon^{2}}+\frac{3}{2 \varepsilon}+3+4 \log (2)-\frac{\pi^{2}}{2}\right] \\
\sigma_{q, g}^{D S} & \sim-\frac{\alpha_{S}}{2 \pi} \frac{(4 \pi)^{\varepsilon}}{\Gamma(1-\varepsilon)} \frac{C_{A}}{2} \int d \Phi_{2}\left(\frac{\mu^{2}}{s_{13}}\right)^{\varepsilon}\left|A_{q \bar{q} g}^{(0)}\right|^{2}\left[\frac{1}{\varepsilon^{2}}+\frac{3}{2 \varepsilon}+3+4 \log (2)-\frac{\pi^{2}}{2}\right] \\
\sigma_{g, q}^{D S} & \sim-\frac{\alpha_{S}}{2 \pi} \frac{(4 \pi)^{\varepsilon}}{\Gamma(1-\varepsilon)} \frac{C_{A}}{2} \int d \Phi_{2}\left(\frac{\mu^{2}}{s_{13}}\right)^{\varepsilon}\left|A_{q \bar{q} g}^{(0)}\right|^{2}\left[\frac{1}{\varepsilon^{2}}+\frac{11}{6 \varepsilon}+\frac{55}{18}+\frac{14}{3} \log (2)-\frac{\pi^{2}}{2}\right] \\
\sigma_{\bar{q}, g}^{D S} & \sim-\frac{\alpha_{S}}{2 \pi} \frac{(4 \pi)^{\varepsilon}}{\Gamma(1-\varepsilon)} \frac{C_{A}}{2} \int d \Phi_{2}\left(\frac{\mu^{2}}{s_{23}}\right)^{\varepsilon}\left|A_{q \bar{q} g}^{(0)}\right|^{2}\left[\frac{1}{\varepsilon^{2}}+\frac{3}{2 \varepsilon}+3+4 \log (2)-\frac{\pi^{2}}{2}\right] \\
\sigma_{g, \bar{q}}^{D S} & \sim-\frac{\alpha_{S}}{2 \pi} \frac{(4 \pi)^{\varepsilon}}{\Gamma(1-\varepsilon)} \frac{C_{A}}{2} \int d \Phi_{2}\left(\frac{\mu^{2}}{s_{23}}\right)^{\varepsilon}\left|A_{q \bar{q} g}^{(0)}\right|^{2}\left[\frac{1}{\varepsilon^{2}}+\frac{11}{6 \varepsilon}+\frac{55}{18}+\frac{14}{3} \log (2)-\frac{\pi^{2}}{2}\right]
\end{aligned}
$$

where $\left|A_{q \bar{q} g}^{(0)}\right|^{2}$ is the Born amplitude for the process $\gamma^{*} \rightarrow q \bar{q} g$ and we have neglected terms of order $\mathcal{O}(\varepsilon)$.

In Figure 23 we show the differential rapidity distribution of the most energetic jet in $e+e-$ annihilation at $\sqrt{s}=125 \mathrm{GeV}$. Jets are clustered using the Durham jet algorithm with resolution parameter set at $y_{\text {cut }}=0.05$. A perfect agreement with the same computation performed using Catani-Seymour dipoles is observed. The same level of agreement is observed for any other differential distribution. 


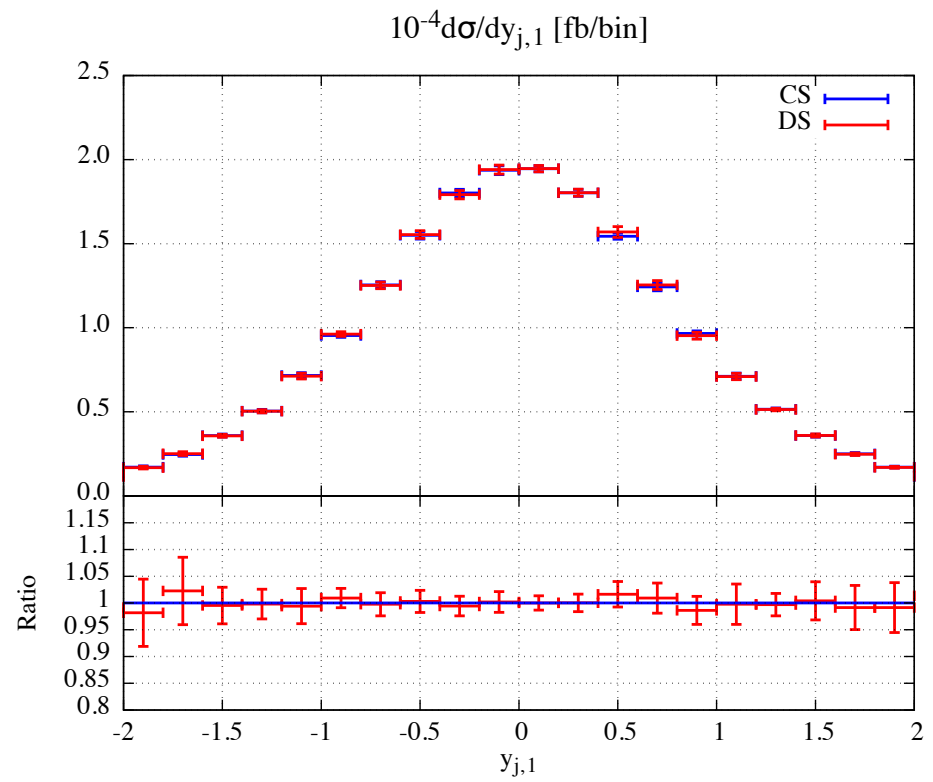

Figure 23. Rapidity distribution of the most energetic jet in $e^{+} e^{-} \rightarrow \gamma^{*} \rightarrow 3$ jets at $\sqrt{s}=125 \mathrm{GeV}$. Jets are clustered using the Durham algorithm with $y_{\text {cut }}=0.05$. Only the NLO corrections are plotted. Results obtained using Dual Subtraction (DS) results are shown in red, while the ones obtained using Catani-Seymour (CS) dipoles are shown in blue. The error bars correspond to the statistical error given by the Monte Carlo integration.

In this way, we have shown that a fully local implementation of an LTD-inspired method for computing cross-sections is reliable. These studies complement the ones presented in Section 8, indicating that the FDU framework constitutes a powerful strategy for reaching higher-order corrections with fully numerical methods. An extension of this technology to calculate $\mathrm{N}^{2} \mathrm{LO}$ corrections for IR-safe observables is being actively studied.

\section{Outlook and Further Developments}

In this manuscript, we reported on the recent developments in the numerical evaluation of multi-loop scattering amplitudes through the application of the Loop-Tree Duality (LTD) formalism. Based on the original approach, at one and two loops [68,70], we presented a novel formulation of LTD [92], which allowed to bootstrap the treatment of Feynman integrals, regardless of the loop order, and shed light to a complete automation. In effect, the decomposition of two- and three-loop scattering amplitudes in terms of dual integrands was obtained by introducing the concepts of maximal (MLT), next-to-maximal (NMLT) and next-to-next-to-maximal ( $\mathrm{N}^{2} \mathrm{MLT}$ ) loop topologies. These families of diagrams share general properties that allow allows for the generalization of the dual representation at any loop order.

Since LTD heavily relies on the application of the Cauchy residue theorem, we studied in detail the treatment of multivariate rational functions in Ref. [97], giving, in this way, a mathematical support to the novel formulation of Ref. [92]. Likewise, we proved all conjectures that were provided in the latter, thus, rendering in a well-organized formalism ready to focus on physical applications. Hence, in view of the LTD decompositions of up to three-loop scattering amplitudes, the natural extension, along the lines of Ref. [92], was considering the four-loop case [95]. In effect, the complexity in the treatment of four-loop topologies increased because, differently from the two- and three-loop cases, this loop order was not described by only one-loop topology, as in the lower cases. Hence, we introduced new configurations that appears at four loops: the $\mathrm{N}^{3} \mathrm{MLT}$ and $\mathrm{N}^{4} \mathrm{MLT}$ families. In this way, our formalism allowed a complete understanding and decomposition of the dual representation of any up to four-loop scattering amplitude. 
In the spirit of profiting from the dual representation of integrands through LTD, we observed that the complete sum of the latter, for a given Feynman integral, led to a representation of an integrand that only manifests physical information. In other words, unphysical singularities or pseudo-thresholds cancel out when all dual integrands are summed up. This is a remarkable feature of LTD that was originally observed in Refs. $[79,87,92]$ and extensively studied in Ref. [96] for MLT, NMLT and $\mathrm{N}^{2}$ MLT configurations. In fact, the work performed in Ref. [96] made a comparison between the numerical stability of dual and causal integrands. Although in the former numerical instabilities, originated from pseudo-thresholds, were present, the latter displayed a smooth behavior: the numerical evaluation was much more stable, and this allowed a straightforward computation of the Feynman integrals. In particular, we considered the numerical integration of ultraviolet finite integrals at three and four loops with presence of several kinematic variables.

The causal representation of scattering amplitudes has recently been a very active topic due to the simple structure of these integrands in terms of causal (physical) thresholds. In fact, an understanding of the structure of the latter started to appear in the literature by means of geometric properties [102] and is yet under consideration. On top of the former approach, it was recently conjectured a breathtaking formulation of the all-loop causal representation of multi-loop scalar integrands, obtained from the features that characterized a topology, vertices and edges [100]. This formulation provides, regardless of the loop order, the most symmetric causal representation of Feynman integrands, in a complementary and independent way to the nested residue calculation used by the LTD formalism. Thus, to give a support to these conjectures, we provide the MATHEMATICA package LOTTY to automate both dual and causal representation of scattering amplitudes [101].

From the above-mentioned discussion, we claim that a treatment of Feynman integrands at an arbitrary number of loops is under control. Moreover, this is not the end of the story when comparing theoretical predictions with data derived by collider experiments. In effect, one yet must deal with ultraviolet (UV) and infrared (IR) singularities. Although the framework at next-to-leading order (NLO) is completely understood by means of the FourDimensional Unsubtraction scheme $[82,84,85]$ and the dual subtractions [86], a clear path towards calculations at higher orders (NNLO and beyond) needs to be devised. Preliminary studies of IR-safe scattering amplitudes has been recently considered in Refs. [79,81]. Moreover, the extension to the calculation of cross-sections at NNLO is under study, in particular, focusing on a careful local treatment of IR singularities.

We expect that the ideas presented in this manuscript will allow detailed elucidation of the state of the art of the LTD formalism and the causal representation of scattering amplitudes by means of the latter. Likewise, the ideas that we have developed will certainly supply the scientific community with a powerful strategy to tackle the calculation of multiloop Feynman integrals, in which phenomenological and formal applications can efficiently be carried out.

Author Contributions: Writing-review and editing, R.J.H.-P. and G.S.; writing-introduction, R.J.H.-P., G.R., G.S. and W.J.T.B.; writing-Section 2, A.E.R.-O. and G.F.R.S.; writing-Section 3, J.d.J.A.-V.; writing-Section 4, J.P.; writing-Sections 5 and 7, G.S.; writing-Section 6, N.S.R.-U.; writing-Section 8, F.D.-M. and R.J.H.-P.; writing-Section 9, R.M.P. and F.T.; writing-conclusions, G.R. and W.J.T.B. All authors have read and agreed to the published version of the manuscript.

Funding: This work is supported by the Spanish Government (Agencia Estatal de Investigación) and ERDF funds from European Commission (Grants No. FPA2017-84445-P), Generalitat Valenciana (Grant No. PROMETEO/2017/053). JP acknowledges support from "la Caixa" Foundation (No. 100010434, LCF/BQ/IN17/11620037), and the European Union's H2020-MSCA Grant Agreement No. 713673; SRU from CONACyT and Universidad Autónoma de Sinaloa; JJAV from Generalitat Valenciana (GRISOLIAP /2018/101); AERO from the Spanish Government (PRE2018085925); and RJHP from the project A1-S-33202 (Ciencia Básica), Ciencia de Frontera 2021-2042 and Sistema Nacional de Investigadores. This article is based upon work from COST Action PARTICLEFACE CA16201, supported by COST (European Cooperation in Science and Technology). https: / / www.cost.eu/ (accessed on 1 June 2021). 
Institutional Review Board Statement: Not applicable.

Informed Consent Statement: Not applicable.

Data Availability Statement: Not applicable.

Acknowledgments: This publication is based upon work from COST Action PARTICLEFACE CA16201, supported by COST (European Cooperation in Science and Technology, https:/ / www.cost. eu/ (accessed on 1 June 2021)).

Conflicts of Interest: The authors declare no conflict of interest.

\section{References}

1. Abada, A.; Abbrescia, M.; AbdusSalam, S.S.; Abdyukhanov, I.; Fernandez, J.A.; Abramov, A.; Aburaia, M.; Acar, A.O.; Adzic, P.R.; Agrawal, P.; et al. FCC Physics Opportunities: Future Circular Collider Conceptual Design Report Volume 1. Eur. Phys. J. C 2019, 79, 474. [CrossRef]

2. Abada, A.; Abbrescia, M.; AbdusSalam, S.S.; Abdyukhanov, I.; Fernandez, J.A.; Abramov, A.; Aburaia, M.; Acar, A.O.; Adzic, P.R.; Agrawal, P.; et al. FCC-ee: The Lepton Collider: Future Circular Collider Conceptual Design Report Volume 2. Eur. Phys. J. ST 2019, 228, 261-623. [CrossRef]

3. Abada, A.; Abbrescia, M.; AbdusSalam, S.S.; Abdyukhanov, I.; Fernandez, J.A.; Abramov, A.; Aburaia, M.; Acar, A.O.; Adzic, P.R.; Agrawal, P.; et al. FCC-hh: The Hadron Collider: Future Circular Collider Conceptual Design Report Volume 3. Eur. Phys. J. ST 2019, 228, 755-1107. [CrossRef]

4. Abada, A.; Abbrescia, M.; AbdusSalam, S.S.; Abdyukhanov, I.; Fernandez, J.A.; Abramov, A.; Aburaia, M.; Acar, A.O.; Adzic, P.R.; Agrawal, P.; et al. HE-LHC: The High-Energy Large Hadron Collider: Future Circular Collider Conceptual Design Report Volume 4. Eur. Phys. J. ST 2019, 228, 1109-1382. [CrossRef]

5. Blondel, A.; Gluza, J.; Jadach, S.; Janot, P.; Riemann, T. (Eds.) Theory for the FCC-ee: Report on the 11th FCC-ee Workshop Theory and Experiments. In vol. 3/2020 of CERN Yellow Reports: Monographs, (Geneva); CERN: Meyrin, Switzerland, 2019. [CrossRef]

6. Bambade, P.; Barklow, T.; Behnke, T.; Berggren, M.; Brau, J.; Burrows, P.; Denisov, D.; Faus-Golfe, A.; Foster, B.; Fujii, K.; et al. The International Linear Collider: A Global Project. arXiv 2019, arXiv:1903.01629.

7. Roloff, P.; Franceschini, R.; Schnoor, U.; Wulzer, A. The Compact Linear $\mathrm{e}^{+} \mathrm{e}^{-}$Collider (CLIC): Physics Potential. arXiv 2018, arXiv:1812.07986.

8. CEPC Conceptual Design Report: Volume 2-Physics \& Detector. arXiv 2018, arXiv:1811.10545.

9. Hooft, G.; Veltman, M.J.G. Regularization and Renormalization of Gauge Fields. Nucl. Phys. 1972, B44, 189-213. [CrossRef]

10. Bollini, C.G.; Giambiagi, J.J. Dimensional Renormalization: The Number of Dimensions as a Regularizing Parameter. Nuovo Cim. 1972, B12, 20-26. [CrossRef]

11. Cicuta, G.M.; Montaldi, E. Analytic renormalization via continuous space dimension. Lett. Nuovo Cim. 1972, 4, 329-332. [CrossRef]

12. Ashmore, J.F. A Method of Gauge Invariant Regularization. Lett. Nuovo Cim. 1972, 4, 289-290. [CrossRef]

13. Gnendiger, C.; Signer, A. $\gamma_{5}$ in the four-dimensional helicity scheme. Phys. Rev. D 2018, 97, 096006. [CrossRef]

14. Bruque, A.M.; Cherchiglia, A.L.; Pérez-Victoria, M. Dimensional regularization vs methods in fixed dimension with and without $\gamma_{5}$. J. High Energy Phys. 2018, 8, 109. [CrossRef]

15. Pittau, R. A four-dimensional approach to quantum field theories. J. High Energy Phys. 2012, 1211, 151. [CrossRef]

16. Fazio, R.A.; Mastrolia, P.; Bobadilla, E.M.W.J.T. On the Four-Dimensional Formulation of Dimensionally Regulated Amplitudes. Eur. Phys. J. 2014, C74, 3197. [CrossRef]

17. Mastrolia, P.; Primo, A.; Schubert, U.; Bobadilla, W.J.T. Off-shell currents and color-kinematics duality. Phys. Lett. 2016, B753, 242-262. [CrossRef]

18. Primo, A.; Bobadilla, W.J.T. BCJ Identities and d-Dimensional Generalized Unitarity. J. High Energy Phys. 2016, 4, 125. [CrossRef]

19. Gnendiger, C.; Signer, A.; Stöckinger, D.; Broggio, A.; Cherchiglia, A.L.; Driencourt-Mangin, F.; Fazio, A.R.; Hiller, B.; Mastrolia, P.; Peraro, T.; et al. To $d$, or not to $d$ : recent developments and comparisons of regularization schemes. Eur. Phys. J. 2017, C77, 471. [CrossRef]

20. Bobadilla, W.J.T.; Sborlini, G.F.R.; Banerjee, P.; Catani, S.; Cherchiglia, A.L.; Cieri, L.; Dhani, P.K.; Driencourt-Mangin, F.; Engel, T.; Ferrera, G.; et al. May the four be with you: Novel IR-subtraction methods to tackle NNLO calculations. arXiv 2020, arXiv:2012.025670.

21. Chetyrkin, K.G.; Tkachov, F.V. Integration by Parts: The Algorithm to Calculate beta Functions in 4 Loops. Nucl. Phys. 1981, B192, 159-204. [CrossRef]

22. Laporta, S. High precision calculation of multiloop Feynman integrals by difference equations. Int. J. Mod. Phys. 2000, A15, 5087-5159. [CrossRef]

23. Binoth, T.; Heinrich, G. An automatized algorithm to compute infrared divergent multiloop integrals. Nucl. Phys. B 2000, 585, 741-759. [CrossRef]

24. Smirnov, A.; Tentyukov, M. Feynman Integral Evaluation by a Sector decomposiTion Approach (FIESTA). Comput. Phys. Commun. 2009, 180, 735-746. [CrossRef] 
25. Carter, J.; Heinrich, G. SecDec: A general program for sector decomposition. Comput. Phys. Commun. 2011, $182,1566-1581$. [CrossRef]

26. Borowka, S.; Heinrich, G.; Jahn, S.; Jones, S.; Kerner, M.; Schlenk, J. pySecDec: A toolbox for the numerical evaluation of multi-scale integrals. Comput. Phys. Commun. 2018, 222, 313-326. [CrossRef]

27. Moriello, F. Generalised power series expansions for the elliptic planar families of Higgs + jet production at two loops. J. High Energy Phys. 2020, 1, 150. [CrossRef]

28. Bonciani, R.; Duca, V.D.; Frellesvig, H.; Henn, J.; Hidding, M.; Maestri, L. Evaluating a family of two-loop non-planar master integrals for Higgs + jet production with full heavy-quark mass dependence. J. High Energy Phys. 2020, 1, 132. [CrossRef]

29. Czakon, M. Tops from Light Quarks: Full Mass Dependence at Two-Loops in QCD. Phys. Lett. B 2008, 664, 307-314. [CrossRef]

30. Heinrich, G. Collider Physics at the Precision Frontier. arXiv 2020, arXiv:2009.00516.

31. Larsen, K.J.; Zhang, Y. Integration-by-parts reductions from unitarity cuts and algebraic geometry. Phys. Rev. D 2016, 93, 041701. [CrossRef]

32. Bern, Z.; Enciso, M.; Ita, H.; Zeng, M. Dual Conformal Symmetry, Integration-by-Parts Reduction, Differential Equations and the Nonplanar Sector. Phys. Rev. D 2017, 96, 096017. [CrossRef]

33. Zeng, M. Differential equations on unitarity cut surfaces. J. High Energy Phys. 2017, 6, 121. [CrossRef]

34. Böhm, J.; Georgoudis, A.; Larsen, K.J.; Schulze, M.; Zhang, Y. Complete sets of logarithmic vector fields for integration-by-parts identities of Feynman integrals.Phys. Rev. D 2018, 98, 025023. [CrossRef]

35. Böhm, J.; Georgoudis, A.; Larsen, K.J.; Schönemann, H.; Zhang, Y. Complete integration-by-parts reductions of the non-planar hexagon-box via module intersections. J. High Energy Phys. 2018, 9, 024. [CrossRef]

36. Bendle, D.; Böhm, J.; Decker, W.; Georgoudis, A.; Pfreundt, F.-J.; Rahn, M.; Wasser, P.; Zhang, Y. Integration-by-parts reductions of Feynman integrals using Singular and GPI-Space. J. High Energy Phys. 2020, 2, 079. [CrossRef]

37. Mastrolia, P.; Ossola, G. On the Integrand-Reduction Method for Two-Loop Scattering Amplitudes. J. High Energy Phys. 2011, 11, 014. [CrossRef]

38. Badger, S.; Frellesvig, H.; Zhang, Y. Hepta-Cuts of Two-Loop Scattering Amplitudes. J. High Energy Phys. $2012,1204,055$. [CrossRef]

39. Zhang, Y. Integrand-Level Reduction of Loop Amplitudes by Computational Algebraic Geometry Methods. J. High Energy Phys. 2012, 9, 042. [CrossRef]

40. Mastrolia, P.; Mirabella, E.; Ossola, G.; Peraro, T. Scattering Amplitudes from Multivariate Polynomial Division. Phys. Lett. 2012, $B 718,173-177$. [CrossRef]

41. Mastrolia, P.; Mirabella, E.; Ossola, G.; Peraro, T. Integrand-Reduction for Two-Loop Scattering Amplitudes through Multivariate Polynomial Division. Phys. Rev. 2013, D87, 085026. [CrossRef]

42. Ita, H. Two-loop Integrand Decomposition into Master Integrals and Surface Terms. Phys. Rev. D 2016, 94, 116015. [CrossRef]

43. Mastrolia, P.; Peraro, T.; Primo, A. Adaptive Integrand Decomposition in parallel and orthogonal space. J. High Energy Phys. 2016, 8, 164. [CrossRef]

44. Ossola, G.; Papadopoulos, C.G.; Pittau, R. Reducing full one-loop amplitudes to scalar integrals at the integrand level. Nucl. Phys. 2007, B763, 147-169. [CrossRef]

45. Baikov, P. Explicit solutions of the three loop vacuum integral recurrence relations. Phys. Lett. B 1996, 385, 404-410. [CrossRef]

46. Frellesvig, H.; Papadopoulos, C.G. Cuts of Feynman Integrals in Baikov representation. J. High Energy Phys. 2017, 04, 083. [CrossRef]

47. Peraro, T. Scattering amplitudes over finite fields and multivariate functional reconstruction. J. High Energy Phys. 2016, 12, 030. [CrossRef]

48. Badger, S.; Brønnum-Hansen, C.; Hartanto, H.B.; Peraro, T. First look at two-loop five-gluon scattering in QCD. Phys. Rev. Lett. 2018, 120, 092001. [CrossRef]

49. Abreu, S.; Cordero, F.F.; Ita, H.; Page, B.; Zeng, M. Planar Two-Loop Five-Gluon Amplitudes from Numerical Unitarity. Phys. Rev. D 2018, 97, 116014. [CrossRef]

50. Badger, S.; Chicherin, D.; Gehrmann, T.; Heinrich, G.; Henn, J.; Peraro, T.; Wasser, P.; Zhang, Y.; Zoia, S. Analytic form of the full two-loop five-gluon all-plus helicity amplitude. Phys. Rev. Lett. 2019, 123, 071601. [CrossRef]

51. Mastrolia, P.; Mizera, S. Feynman Integrals and Intersection Theory. J. High Energy Phys. 2019, 2, 139. [CrossRef]

52. Frellesvig, H.; Gasparotto, F.; Laporta, S.; Mandal, M.K.; Mastrolia, P.; Mattiazzi, L.; Mizera, S. Decomposition of Feynman Integrals on the Maximal Cut by Intersection Numbers. J. High Energy Phys. 2019, 5, 153. [CrossRef]

53. Frellesvig, H.; Gasparotto, F.; Mandal, M.K.; Mastrolia, P.; Mattiazzi, L.; Mizera, S. Vector Space of Feynman Integrals and Multivariate Intersection Numbers. Phys. Rev. Lett. 2019, 123, 201602. [CrossRef]

54. Weinzierl, S. On the computation of intersection numbers for twisted cocycles. arXiv 2020, arXiv:2002.01930.

55. Kunszt, Z.; Soper, D.E. Calculation of jet cross-sections in hadron collisions at order alpha-s**3. Phys. Rev. 1992, D46, 192-221. [CrossRef]

56. Frixione, S.; Kunszt, Z.; Signer, A. Three jet cross-sections to next-to-leading order. Nucl. Phys. 1996, B467, 399-442. [CrossRef]

57. Catani, S.; Seymour, M. The Dipole formalism for the calculation of QCD jet cross-sections at next-to-leading order. Phys. Lett. 1996, B378, 287-301. [CrossRef] 
58. Catani, S.; Seymour, M. A General algorithm for calculating jet cross-sections in NLO QCD. Nucl. Phys. 1997, B485, 291-419. [CrossRef]

59. Catani, S.; de Florian, D.; Grazzini, M. Universality of nonleading logarithmic contributions in transverse momentum distributions. Nucl. Phys. B 2001, 596, 299-312. [CrossRef]

60. Catani, S.; Cieri, L.; de Florian, D.; Ferrera, G.; Grazzini, M. Universality of transverse-momentum resummation and hard factors at the NNLO. Nucl. Phys. B 2014, 881, 414-443. [CrossRef]

61. Ridder, A.Ge.; Gehrmann, T.; Glover, E.W.N. Antenna subtraction at NNLO. J. High Energy Phys. 2005, 9, 056. [CrossRef]

62. Kardos, A. Jet production in the CoLoRFulNNLO framework. In Proceedings of the 12th International Symposium on Radiative Corrections (RADCOR 2015) and LoopFest XIV (Radiative Corrections for the LHC and Future Colliders)(RADCOR2015), Los Angeles, CA, USA, 15-19 June 2015. [CrossRef]

63. Duca, V.D.; Duhr, C.; Kardos, A.; Somogyi, G.; Szőr, Z.; Trócsányi, Z. Jet production in the CoLoRFulNNLO method: Event shapes in electron-positron collisions. Phys. Rev. D 2016, 94, 074019. [CrossRef]

64. Gaunt, J.; Stahlhofen, M.; Walsh, F.J.T.J.R. N-jettiness Subtractions for NNLO QCD Calculations. J. High Energy Phys. 2015, 9, 058 [CrossRef]

65. Boughezal, R.; Caola, F.; Melnikov, K.; Petriello, F.; Schulze, M. Higgs boson production in association with a jet at next-to-next-toleading order. Phys. Rev. Lett. 2015, 115, 082003. [CrossRef] [PubMed]

66. Magnea, L.; Maina, E.; Pelliccioli, G.; Signorile-Signorile, C.; Torrielli, P.; Uccirati, S. Local analytic sector subtraction at NNLO. J. High Energy Phys. 2018 12, 107. [CrossRef]

67. Magnea, L.; Maina, E.; Pelliccioli, G.; Signorile-Signorile, C.; Torrielli, P.; Uccirati, S. Local analytic sector subtraction for final state radiation at NNLO. PoS 2019, RADCOR2019, 057. [CrossRef]

68. Catani, S.; Gleisberg, T.; Krauss, F.; Rodrigo, G.; Winter, J.-C. From loops to trees by-passing Feynman's theorem. J. High Energy Phys. 2008, 9, 065. [CrossRef]

69. Rodrigo, G.; Catani, S.; Gleisberg, T.; Krauss, F.; Winter, J.-C. From multileg loops to trees (by-passing Feynman's Tree Theorem). Nucl. Phys. B Proc. Suppl. 2008, 183, 262-267. [CrossRef]

70. Bierenbaum, I.; Catani, S.; Draggiotis, P.; Rodrigo, G. A Tree-Loop Duality Relation at Two Loops and Beyond. J. High Energy Phys. 2010, 10, 073. [CrossRef]

71. Bierenbaum, I.; Buchta, S.; Draggiotis, P.; Malamos, I.; Rodrigo, G. Tree-Loop Duality Relation beyond simple poles. J. High Energy Phys. 2013, 3, 025. [CrossRef]

72. Buchta, S.; Chachamis, G.; Draggiotis, P.; Malamos, I.; Rodrigo, G. On the singular behaviour of scattering amplitudes in quantum field theory. J. High Energy Phys. 2014, 11, 014. [CrossRef]

73. Buchta, S. Theoretical Foundations and Applications of the Loop-Tree Duality in Quantum Field Theories. Ph.D. Thesis, University of Valencia, Valencia, Spain, 2015.

74. Buchta, S.; Chachamis, G.; Draggiotis, P.; Rodrigo, G. Numerical implementation of the loop-tree duality method. Eur. Phys. J. 2017, C77, 274. [CrossRef]

75. Driencourt-Mangin, F.; Rodrigo, G.; Sborlini, G.F. Universal dual amplitudes and asymptotic expansions for $g g \rightarrow H$ and $H \rightarrow \gamma \gamma$ in four dimensions. Eur. Phys. J. C 2018, 78, 231. [CrossRef]

76. Plenter, J. Asymptotic Expansions Through the Loop-Tree Duality. Acta Phys. Polon. B 2019, 50, 1983-1992. [CrossRef]

77. Plenter, J.; Rodrigo, G. Asymptotic expansions through the loop-tree duality. arXiv 2020, arXiv:2005.02119.

78. Jurado, J.L.; Rodrigo, G.; Bobadilla, W.J.T. From Jacobi off-shell currents to integral relations. J. High Energy Phys. 2017, 12, 122. [CrossRef]

79. $\quad$ Driencourt-Mangin, F.; Rodrigo, G.; Sborlini, G.F.R.; Bobadilla, W.J.T. Universal four-dimensional representation of $H \rightarrow \gamma \gamma$ at two loops through the Loop-Tree Duality. J. High Energy Phys. 2019, 2, 143. [CrossRef]

80. Driencourt-Mangin, F. Four-Dimensional Representation of Scattering Amplitudes and Physical Observables through the Application of the Loop-Tree Duality Theorem. Ph.D. Thesis, University of Valencia, Valencia, Spain, 2019.

81. Driencourt-Mangin, F.; Rodrigo, G.; Sborlini, G.F.; Torres Bobadilla, W.J. On the interplay between the loop-tree duality and helicity amplitudes. arXiv 2019, arXiv:1911.11125.

82. Hernandez-Pinto, R.J.; Sborlini, G.F.R.; Rodrigo, G. Towards gauge theories in four dimensions. J. High Energy Phys. 2016, 2, 044. [CrossRef]

83. Sborlini, G.F. Loop-tree duality and quantum field theory in four dimensions. PoS 2016, RADCOR2015, 082. [CrossRef]

84. Sborlini, G.F.R.; Driencourt-Mangin, F.; Hernandez-Pinto, R.; Rodrigo, G. Four-dimensional unsubtraction from the loop-tree duality. J. High Energy Phys. 2016, 8, 160. [CrossRef]

85. Sborlini, G.F.R.; Driencourt-Mangin, F.; Rodrigo, G. Four-dimensional unsubtraction with massive particles. J. High Energy Phys. 2016, 10, 162. [CrossRef]

86. Prisco, R.M.; Tramontano, F. Dual Subtractions. arXiv 2020, arXiv:2012.05012.

87. Aguilera-Verdugo, J.J.; Driencourt-Mangin, F.; Plenter, J.; Ramírez-Uribe, S.; Rodrigo, G.; Sborlini, G.F.; Bobadilla, W.J.T.; Tracz, S. Causality, unitarity thresholds, anomalous thresholds and infrared singularities from the loop-tree duality at higher orders. J. High Energy Phys. 2019, 12, 163. [CrossRef]

88. Cutkosky, R.E. Singularities and discontinuities of Feynman amplitudes. J. Math. Phys. 1960, 1, 429-433. [CrossRef]

89. Tomboulis, E. Causality and Unitarity via the Tree-Loop Duality Relation. J. High Energy Phys. 2017, 5, 148. [CrossRef] 
90. Runkel, R.; Szőr, Z.; Vesga, J.P.; Weinzierl, S. Causality and loop-tree duality at higher loops. Phys. Rev. Lett. 2019, 122, 111603. [CrossRef] [PubMed]

91. Runkel, R.; Szőr, Z.; Vesga, J.P.; Weinzierl, S. Integrands of loop amplitudes within loop-tree duality. Phys. Rev. D 2020, 101, 116014. [CrossRef]

92. Aguilera-Verdugo, J.J.; Driencourt-Mangin, F.; Pinto, R.J.H.; Plenter, J.; Ramirez-Uribe, S.; Olivo, A.E.R.; Rodrigo, G.; Sborlini, G.F.; Bobadilla, W.J.T.; Tracz, S. Open loop amplitudes and causality to all orders and powers from the loop-tree duality. Phys. Rev. Lett. 2020, 124, 211602. [CrossRef] [PubMed]

93. Capatti, Z.; Hirschi, V.; Kermanschah, D.; Pelloni, A.; Ruijl, B. Numerical Loop-Tree Duality: Contour deformation and subtraction. J. High Energy Phys. 2020, 4, 096. [CrossRef]

94. Capatti, Z.; Hirschi, V.; Kermanschah, D.; Ruijl, B. Loop-Tree Duality for Multiloop Numerical Integration. Phys. Rev. Lett. 2019, 123, 151602. [CrossRef]

95. Ramírez-Uribe, S.; Hernández-Pinto, R.J.; Rodrigo, G.; Sborlini, G.F.R.; Bobadilla, W.J.T. Universal opening of four-loop scattering amplitudes to trees. J. High Energy Phys. 2021, 4, 129. [CrossRef]

96. Aguilera-Verdugo, J.J.; Hernandez-Pinto, R.J.; Rodrigo, G.; Sborlini, G.F.R.; Bobadilla, W.J.T. Causal representation of multi-loop Feynman integrands within the loop-tree duality. J. High Energy Phys. 2021, 1, 069. [CrossRef]

97. Aguilera-Verdugo, J.J.; Hernández-Pinto, R.J.; Rodrigo, G.; Sborlini, G.F.R.; Bobadilla, W.J.T. Mathematical properties of nested residues and their application to multi-loop scattering amplitudes. J. High Energy Phys. 2021, 2, 112. [CrossRef]

98. Aguilera-Verdugo, J.; Hernández-Pinto, R.; Ramírez-Uribe, S.; Rodrigo, G.; Sborlini, G.; Torres Bobadilla, W.J. Manifestly Causal Scattering Amplitudes. Letter of Intention submitted to SnowMass 2021. Available online: https://www.snowmass21.org/docs/ files/summaries/TF/SNOWMASS21-TF4_TF6_TorresBobadilla-093.pdf (accessed on 1 June 2021).

99. Capatti, Z.; Hirschi, V.; Kermanschah, D.; Pelloni, A.; Ruijl, B. Manifestly Causal Loop-Tree Duality. arXiv 2020, arXiv:2009.05509.

100. Bobadilla, W.J.T. Loop-tree duality from vertices and edges. J. High Energy Phys. 2021, 04, 183. [CrossRef]

101. Bobadilla, W.J.T. Lotty-The loop-tree duality automation. arXiv 2021, arXiv:2103.09237.

102. Sborlini, G.F.R. A geometrical approach to causality in multi-loop amplitudes. arXiv 2021, arXiv:2102.05062.

103. Ramírez-Uribe, S.; Rentería-Olivo, A.; Rodrigo, G.; Sborlini, G.; Silva, L.V. Quantum algorithm for Feynman loop integrals. arXiv 2021, arXiv:2105.08703.

104. Beneke, M.; Smirnov, V.A. Asymptotic expansion of Feynman integrals near threshold. Nucl. Phys. 1998, B522, 321-344. [CrossRef]

105. Pak, A.; Smirnov, A. Geometric approach to asymptotic expansion of Feynman integrals. Eur. Phys. J. C 2011, 71, 1626. [CrossRef]

106. Jantzen, B. Foundation and generalization of the expansion by regions. J. High Energy Phys. 2011, 12, 076. [CrossRef]

107. Mishima, G. High-Energy Expansion of Two-Loop Massive Four-Point Diagrams. J. High Energy Phys. 2019, 2, 080. [CrossRef]

108. Semenova, T.Y.; Smirnov, A.V.; Smirnov, V.A. On the status of expansion by regions. Eur. Phys. J. C 2019, 79, 136. [CrossRef]

109. Peraro, T. FiniteFlow: Multivariate functional reconstruction using finite fields and dataflow graphs. J. High Energy Phys. 2019, 7, 031. [CrossRef]

110. Von Manteuffel, A.; Schabinger, R.M. A novel approach to integration by parts reduction. Phys. Lett. B 2015, 744, 101-104. [CrossRef]

111. Sborlini, G.F.R.; Hernández-Pinto, R.; Rodrigo, G. From dimensional regularization to NLO computations in four dimensions. PoS 2015, EPS-HEP2015, 479. [CrossRef]

112. Nogueira, P. Automatic Feynman graph generation. J. Comput. Phys. 1993, 105, 279-289. [CrossRef]

113. Kinoshita, T. Mass singularities of Feynman amplitudes. J. Math. Phys. 1962, 3, 650-677. [CrossRef]

114. Lee, T.D.; Nauenberg, M. Degenerate Systems and Mass Singularities. Phys. Rev. 1964, 133, B1549-B1562. [CrossRef]

115. Kosower, D.A.; Uwer, P. One loop splitting amplitudes in gauge theory. Nucl. Phys. B 1999, 563, 477-505. [CrossRef]

116. Sborlini, G.F.R.; de Florian, D.; Rodrigo, G. Double collinear splitting amplitudes at next-to-leading order. J. High Energy Phys. 2014, 1, 018. [CrossRef]

117. Catani, S.; Grazzini, M. Infrared factorization of tree level QCD amplitudes at the next-to-next-to-leading order and beyond. Nucl. Phys. B 2000, 570, 287-325. [CrossRef]

118. Catani, S.; de Florian, D.; Rodrigo, G. The Triple collinear limit of one loop QCD amplitudes. Phys. Lett. B 2004, 586, 323-331. [CrossRef]

119. Sborlini, G.F.R. Comportamiento Singular de las Amplitudes de Scattering en el Límite Colineal. Ph.D. Thesis, University of Buenos Aires, Buenos Aires, Argentina, 2014.

120. Sborlini, G.F.R.; de Florian, D.; Rodrigo, G. Triple collinear splitting functions at NLO for scattering processes with photons. J. High Energy Phys. 2014, 10, 161. [CrossRef]

121. Sborlini, G.F.R.; de Florian, D.; Rodrigo, G. Polarized triple-collinear splitting functions at NLO for processes with photons. J. High Energy Phys. 2015, 3, 021. [CrossRef]

122. Duca, V.D.; Duhr, C.; Haindl, R.; Lazopoulos, A.; Michel, M. Tree-level splitting amplitudes for a quark into four collinear partons. J. High Energy Phys. 2020, 2, 189. [CrossRef]

123. Duca, V.D.; Duhr, C.; Haindl, R.; Lazopoulos, A.; Michel, M. Tree-level splitting amplitudes for a gluon into four collinear partons. J. High Energy Phys. 2020, 10, 093. [CrossRef] 